\title{
Characterization of interpolation between Grand, small or classical Lebesgue spaces
}

\author{
Alberto Fiorenza \\ Dipartimento di Architettura, Università di Napoli "Federico II", via Monteoliveto, 3, I-80134 Napoli, ITALY, \\ and Istituto per le Applicazioni del Calcolo "Mauro Picone" \\ Consiglio Nazionale delle Ricerche via Pietro Castellino, 111 I-80131Napoli, ITALY \\ e-mail: fiorenza@unina.it \\ Maria Rosaria Formica \\ Università degli Studi di Napoli "Parthenope", via Generale Parisi 13, 80132, Napoli, ITALY \\ e-mail: mara.formica@uniparthenope.it
}

Amiran Gogatishvili

Institute of Mathematics of the Czech Academy of Sciences - Žitná, 11567 Prague 1, CZECH REPUBLIC e-mail: gogatish@math.cas.cz

Tengiz Kopaliani

Faculty of Exact and Natural Sciences

Javakhishvili Tbilisi State University - University St. 20143 Tbilisi, GEORGIA

e-mail: tengiz.kopaliani@tsu.ge

\section{Jean Michel Rakotoson*}

Laboratoire de Mathématiques et Applications - Université de Poitiers,

Avenue Marie et Pierre Curie,Téléport 2,BP 30179,86692 Futuroscope Chasseneuil Cedex, FRANCE

rako@math.univ-poitiers.fr jean.michel.rakotoson@univ-poitiers.fr

August 4, 2018

\footnotetext{
*corresponding author: rako@math.univ-poitiers.fr, jean.michel.rakotoson@univ-poitiers.fr
} 
In this paper, we show that the interpolation spaces between Grand, small or classical Lebesgue are so called Lorentz-Zygmund spaces or more generally $G \Gamma$-spaces. As a direct consequence of our results any Lorentz-Zygmund space $L^{a, r}(\log L)^{\beta}$, is an interpolation space in the sense of Peetre between either two Grand Lebesgue spaces or between two small spaces provided that $1<a<\infty, \beta \neq 0$. The method consists in computing the so called K-functional of the interpolation space and in identifying the associated norm.

\section{Contents}

1 Introduction. Main results

2 Notations. Preliminary Lemmas

3 Computation of some $K$-functionals and characterization of the interpolation spaces $\left(L^{p), \alpha}, L^{q), \alpha}\right)_{\theta, r}$

4 Small Lebesgue space as interpolation of usual Lebesgue spaces

5 Interpolation between small, Grand Lebesgue spaces and the associated $K$-functional

6 The critical case $p=q$. The interpolation space $\left(L^{p)}, L^{(p)}\right.$ and its $K$ functional

\section{Introduction. Main results}

Let $\left(X_{0},\|\cdot\|_{0}\right),\left(X_{1},\|\cdot\|_{1}\right)$ two Banach spaces contained continuously in a Hausdorff topological vector space (that is $\left(X_{0}, X_{1}\right)$ is a compatible couple).

For $g \in X_{0}+X_{1}, t>0$ one defines the so called $K$ functional $K\left(g, t ; X_{0}, X_{1}\right) \doteq K(g, t)$ by setting

$$
K(g, t)=\inf _{g=g_{0}+g_{1}}\left(\left\|g_{0}\right\|_{0}+t\left\|g_{1}\right\|_{1}\right) .
$$

For $0 \leqslant \theta \leqslant 1,1 \leqslant p \leqslant+\infty, \alpha \in \mathbb{R}$ we shall consider

$$
\left(X_{0}, X_{1}\right)_{\theta, p ; \alpha}=\left\{g \in X_{0}+X_{1},\|g\|_{\theta, p ; \alpha}=\left\|t^{-\theta-\frac{1}{p}}(1-\log t)^{\alpha} K(g, t)\right\|_{L^{p}(0,1)} \text { is finite }\right\} .
$$

Here $\|\cdot\|_{V}$ denotes the norm in a Banach space $V$. The weighted Lebesgue space $L^{p}(0,1 ; \omega), 0<p \leqslant+\infty$ is endowed with the usual norm or quasi norm, where $\omega$ is a weight function on $(0,1)$. 
Our definition of the interpolation space is different from the usual one (see [5, 22]) since we restrict the norms on the interval $(0,1)$.

If we consider ordered couple, i.e. $X_{1} \hookrightarrow X_{0}$ and $\alpha=0,\left(X_{0}, X_{1}\right)_{\theta, p ; 0}=\left(X_{0}, X_{1}\right)_{\theta, p}$ is the interpolation space as it is defined by J. Peetre (see [5, 22, 6]).

A particular attention will be brought to the so-called Grand Lebesgue space $L^{p), \alpha}(\Omega)$, with $\Omega$ a bounded (open) set of $\mathbb{R}^{n}$ whose measure is $1,1<p<+\infty, \alpha>0$ defined as $L^{p), \alpha}(\Omega)=\left\{f: \Omega \rightarrow \mathbb{R}\right.$ measurable, $\left.\|f\|_{p), \alpha}=\sup _{0<t<1}(1-\log t)^{-\frac{\alpha}{p}}\left(\int_{t}^{1} f_{*}^{p}(\sigma) d \sigma\right)^{\frac{1}{p}}<+\infty\right\}$ and its associated spaces $L^{\left(p^{\prime}, \alpha\right.}(\Omega), \frac{1}{p^{\prime}}+\frac{1}{p}=1$ defined as (see [12])

$L^{\left(p^{\prime}, \alpha\right.}(\Omega)=\left\{f: \Omega \rightarrow \mathbb{R}\right.$ measurable, $\left.\|f\|_{\left(p^{\prime}, \alpha\right.}=\int_{0}^{1}(1-\log t)^{-\frac{\alpha}{p^{\prime}}+\alpha-1}\left(\int_{0}^{t} f_{*}^{p^{\prime}}(\sigma) d \sigma\right)^{\frac{1}{p^{\prime}}} \frac{d t}{t}<+\infty\right\}$.

Here, $f_{*}$ is the decreasing rearrangement of $|f|$, say it is the generalized inverse of the distribution function

$$
D_{f}(t)=\text { measure }\{x \in \Omega,|f(x)|>t\}, t \in \mathbb{R}_{+} .
$$

Many works related to Grand and small Lebesgue spaces have been recently done (see for instance [2, 12, 14, 15, 9]).

We will show in particular, the

\section{Theorem 1.1.}

Let $1<p<q, \alpha>0$. Then

$$
L^{q), \alpha}=\left(L^{p), \alpha}, L^{q}\right)_{1, \infty ;-\frac{\alpha}{q}} .
$$

An explicit equivalent of $K\left(f, t ; L^{p), \alpha}, L^{q}\right)$ is given in Theorem 3.2 .

For convenience, we will sometimes drop the dependence with respect to the domain $\Omega$ or $(0,1)$ and we shall write $L^{p}(\Omega)=L^{p} \ldots$ etc

More, we will write sometimes $\int_{E} f_{*}^{m}(t) d t=\int_{E} f_{*}^{m}$.

Definition 1.1. (Lorentz-Zygmund space)

For $1 \leqslant p, q \leqslant \infty,-\infty<\alpha<+\infty$, the Lorentz-Zygmund space $L^{p, q}(\log L)^{\alpha}$ consists 
of all functions $f$ measurable such that

$$
\|f\|_{p, q ; \alpha}= \begin{cases}\left(\int_{0}^{1}\left[t^{\frac{1}{p}-\frac{1}{q}}(1-\log t)^{\alpha} f_{*}(t)\right]^{q} d t\right)^{\frac{1}{q}} & \text { if } 1 \leqslant q<+\infty, \\ \sup _{0<t<1} t^{\frac{1}{p}}(1-\log t)^{\alpha} f_{*}(t) & \text { if } q=+\infty\end{cases}
$$

One of the major theorems of the first section will be

\section{Theorem 1.2.}

Let $0<\theta<1,1 \leqslant r<+\infty, \alpha>0,1<p<q$. Then

$$
\left(L^{p), \alpha}, L^{q), \alpha}\right)_{\theta, r}=L^{p_{\theta}, r}(\log L)^{-\frac{\alpha}{p_{\theta}}} \quad \text { where } \frac{1}{p_{\theta}}=\frac{1-\theta}{p}+\frac{\theta}{q} .
$$

We will also use the following extension of Generalized Gamma space (see [17]).

\section{Definition 1.2. of a Generalized Gamma space with double weights}

Let $w_{1}, w_{2}$ be two weights on $(0,1), m \in[1,+\infty], 1 \leqslant p<+\infty$. We assume the following conditions:

c1) There exists $K_{12}>0$ such that $w_{2}(2 t) \leqslant K_{12} w_{2}(t) \forall t \in(0,1 / 2)$. The space $L^{p}\left(0,1 ; w_{2}\right)$ is continuously embedded in $L^{1}(0,1)$.

c2) The function $\int_{0}^{t} w_{2}(\sigma) d \sigma$ belongs to $L^{\frac{m}{p}}\left(0,1 ; w_{1}\right)$.

A generalized Gamma space with double weights is the set:

$$
G \Gamma\left(p, m ; w_{1}, w_{2}\right)=\left\{v: \Omega \rightarrow \mathbb{R} \text { measurable } \int_{0}^{t} v_{*}^{p}(\sigma) w_{2}(\sigma) d \sigma \text { is in } L^{\frac{m}{p}}\left(0,1 ; w_{1}\right)\right\} .
$$

\section{Property 1.1.}

Let $G \Gamma\left(p, m ; w_{1}, w_{2}\right)$ be a Generalized Gamma space with double weights and let us define for $v \in G \Gamma\left(p, m ; w_{1}, w_{2}\right)$

$$
\rho(v)=\left[\int_{0}^{1} w_{1}(t)\left(\int_{0}^{t} v_{*}^{p}(\sigma) w_{2}(\sigma) d \sigma\right)^{\frac{m}{p}} d t\right]^{\frac{1}{m}}
$$

with the obvious change for $m=+\infty$.

Then,

1. $\rho$ is a quasinorm. 
2. $G \Gamma\left(p, m ; w_{1}, w_{2}\right)$ endowed with $\rho$ is a quasi-Banach function space.

3. If $w_{2}=1$

$$
G \Gamma\left(p, m ; w_{1}, 1\right)=G \Gamma\left(p, m ; w_{1}\right)
$$

\section{Proof:}

1. Due to the property of the monotone rearrangement we have $\rho(v)=0$ if and only if $v=0$ and for $\lambda \in \mathbb{R} \rho(\lambda v)=|\lambda| \rho(v)$. Let us show that

$$
\rho\left(v_{1}+v_{2}\right) \leqslant\left(2 K_{12}\right)^{\frac{1}{p}}\left(\rho\left(v_{1}\right)+\rho\left(v_{2}\right)\right) \text { for } v_{1} \text { and } v_{2} \text { in } G \Gamma\left(p, m ; w_{1}, w_{2}\right) .
$$

We have for all $\sigma \in(0,1), v=v_{1}+v_{2}$

$$
\left(v_{1}+v_{2}\right)_{*}(\sigma) \leqslant v_{1 *}\left(\frac{\sigma}{2}\right)+v_{2 *}\left(\frac{\sigma}{2}\right) .
$$

Therefore, we have (using the triangle inequality)

$$
\begin{aligned}
\left(\int_{0}^{t} v_{*}^{p}(\sigma) w_{2}(\sigma) d \sigma\right)^{\frac{1}{p}} & \leqslant\left(\int_{0}^{t} v_{1 *}^{p}\left(\frac{\sigma}{2}\right) w_{2}(\sigma) d \sigma\right)^{\frac{1}{p}}+\left(\int_{0}^{t} v_{2 *}^{p}\left(\frac{\sigma}{2}\right) w_{2}(\sigma) d \sigma\right)^{\frac{1}{p}}(2) \\
& \leqslant 2^{\frac{1}{p}}\left(\int_{0}^{\frac{t}{2}} v_{1 *}^{p}(s) w_{2}(2 s) d s\right)^{\frac{1}{p}}+2^{\frac{1}{p}}\left(\int_{0}^{\frac{t}{2}} v_{2 *}^{p}(s) w_{2}(2 s) d s\right)^{\frac{1}{p}}
\end{aligned}
$$

Using condition $\mathrm{c} 1$ ), we have

$$
\left(\int_{0}^{t} v_{*}^{p}(\sigma) w_{2}(\sigma) d \sigma\right)^{\frac{1}{p}} \leqslant\left(2 K_{12}\right)^{\frac{1}{p}}\left[\left(\int_{0}^{t} v_{1 *}^{p}(s) w_{2}(s) d s\right)^{\frac{1}{p}}+\left(\int_{0}^{t} v_{2 *}^{p}(s) w_{2}(s) d s\right)^{\frac{1}{p}}\right] .
$$

Using again the triangle inequality with the space $L^{m}\left(0,1 ; w_{1}\right)$ we derive that:

$$
\rho\left(v_{1}+v_{2}\right) \leqslant\left(2 K_{12}\right)^{\frac{1}{p}}\left[\rho\left(v_{1}\right)+\rho\left(v_{2}\right)\right] .
$$

2. The function $\rho$ satisfies:

If $0 \leqslant v_{1} \leqslant v_{2}$ then $v_{1 *} \leqslant v_{2 *}$ everywhere on $(0,1)$ so that

$$
\rho\left(v_{1}\right) \leqslant \rho\left(v_{2}\right) \text {. }
$$

If $0 \leqslant v_{1 k} \nearrow v$ almost-everywhere then by the Beppo-Levi's theorem we have for all $t \in[0,1]$

$$
\lim _{k \rightarrow+\infty}\left(\int_{0}^{t} v_{1 k *}^{p}(\sigma) w_{2}(\sigma) d \sigma\right)^{\frac{1}{p}}=\left(\int_{0}^{t} v_{*}^{p}(\sigma) w_{2}(\sigma) d \sigma\right)^{\frac{1}{p}}
$$


since $v_{1 k *} \nearrow v_{*}$ everywhere on $[0,1]$.

Because $L^{m}\left(0,1 ; w_{1}\right)$ is a Banach function space, we deduce that

$$
\lim _{k \rightarrow+\infty} \rho\left(v_{1 k}\right)=\rho(v)
$$

The condition c2) implies that

$$
\rho\left(\chi_{E}\right) \leqslant \rho(1)<+\infty
$$

for any $E \subset \Omega, \chi_{E}$ denoting its characteristic function.

To conclude that it is a complete space, we shall prove the inequality in next Proposition 1.1, which has been already given in the frame of $G \Gamma\left(p, m ; w_{1}\right)$ (with one weight (see [16, 17, 14])).

\section{Proposition 1.1. Inequality for $G \Gamma$}

Let $v \in G \Gamma\left(p, m ; w_{1}, w_{2}\right), E \subset \Omega,|E|>0$. Then

$$
\frac{\rho(v)\left[\int_{0}^{|E|} w_{2}(\sigma) d \sigma\right]^{\frac{1}{p}}}{\left[\int_{0}^{|E|} w_{1}(t)\left(\int_{0}^{t} w_{2}(s) d s\right)^{\frac{m}{p}} d t\right]^{\frac{1}{m}}} \geqslant\left[\int_{0}^{|E|} v_{*}^{p}(\sigma) w_{2}(\sigma) d \sigma\right]^{\frac{1}{p}} .
$$

\section{Proof:}

Let us set

$$
V(t)=\frac{\int_{0}^{t} v_{*}^{p}(\sigma) w_{2}(\sigma) d \sigma}{\int_{0}^{t} w_{2}(\sigma) d \sigma} .
$$

Then $V$ is decreasing since $v_{*}^{p}$ is decreasing. Therefore, we have for $1 \leqslant m<+\infty$

$\rho(v)=\left[\int_{0}^{1} V^{\frac{m}{p}}(t) w_{1}(t)\left(\int_{0}^{t} w_{2}(\sigma) d \sigma\right)^{\frac{m}{p}} d t\right]^{\frac{1}{m}} \geqslant V^{\frac{1}{p}}(|E|)\left[\int_{0}^{|E|} w_{1}(t)\left(\int_{0}^{t} w_{2}(\sigma) d \sigma\right)^{\frac{m}{p}} d t\right]^{\frac{1}{m}}$.

This gives the inequality $m<+\infty$. 
For $m=+\infty$, the argument is the same, since

$$
\begin{aligned}
\sup _{t \leqslant 1} w_{1}(t)\left(\int_{0}^{t} v_{*}^{p}(\sigma) w_{2}(\sigma) d \sigma\right)^{\frac{1}{p}} & \geqslant \sup _{t \leqslant|E|} V^{\frac{1}{p}}(t) w_{1}(t)\left(\int_{0}^{t} w_{2}(\sigma) d \sigma\right)^{\frac{1}{p}} \\
& \geqslant V^{\frac{1}{p}}(|E|) \sup _{t \leqslant|E|} w_{1}(t)\left(\int_{0}^{t} w_{2}(\sigma) d \sigma\right)^{\frac{1}{p}} .
\end{aligned}
$$

One of our results which motivates the introduction of Generalized Gamma space with double weights is

\section{Theorem 1.3.}

Let $1<p<+\infty, 0<\theta<1,1 \leqslant r<+\infty$. Then

$\left(L^{p)}, L^{(p}\right)_{\theta, r}=G \Gamma\left(p, r ; w_{1}, w_{2}\right)$ with $w_{1}(t)=t^{-1}(1-\log t)^{\theta r-1}, w_{2}(t)=(1-\log t)^{-1}, t \in(0,1)$.

The proof is given in the last theorem of this paper.

\section{Notations. Preliminary Lemmas}

For two positive quantities $A$ and $B$ depending on some parameters like functions (real number as a function on $(0,1))$ we shall write $A \lesssim B$ if there exists a constant $c>0$ independent of the parameters such that $A \leqslant c B$, and $A \approx B$ if $B \lesssim A$ and $A \lesssim B$.

\section{Proposition 2.1.}

Let $a>0, b \in \mathbb{R}, \Phi(t)=t^{a}(1-\log t)^{b}, t \in(0,1)$. Then, there exists an invertible function $\varphi$ from $[0,1]$ into $[0,1]$ such that

1. $t \approx \Phi(\varphi(t))=\varphi(t)^{a}(1-\log \varphi(t))^{b}$

2. $1+|\log \varphi(t)| \approx 1+|\log t|$ for $t \in(0,1)$.

\section{Proof:}

Let us set $t_{0}=\left\{\begin{array}{ll}e^{\frac{a-b}{a}} & \text { if } a<b, \\ 1 & \text { if } a \geqslant b .\end{array}\right.$ Then $\Phi$ is strictly increasing on $\left[0, t_{0}\right]$.

Define $g(t)=\frac{\Phi\left(t t_{0}\right)}{\Phi\left(t_{0}\right)}$ for $t \in[0,1]$. Then $g$ is continuous and strictly increasing from $[0,1]$ into itself, and $g(t) \approx t^{a}(1-\log t)^{b}$ and then $1-\log g(t) \approx 1-\log t$. Setting $\varphi(t)=g^{-1}(t)$ the inverse of $g$ we have the result. 


\section{Proposition 2.2.}

Let $\beta \in \mathbb{R},-\infty<\alpha<1$. Then, there exists $c_{\alpha \beta}>0$ :

$$
\int_{0}^{a} t^{-\alpha}(1-\log t)^{\beta} d t \leqslant c_{\alpha \beta} a^{1-\alpha}(1-\log a)^{\beta} \quad \forall a \in[0,1] .
$$

Moreover, if $\beta \geqslant 0$ then

$$
\int_{0}^{a} t^{-\alpha}(1-\log t)^{\beta} d t \geqslant \frac{1}{1-\alpha} a^{1-\alpha}(1-\log a)^{\beta} .
$$

\section{Proof:}

We start with the case $\beta>0$.

Let $k \in \mathbb{N}$ such $k-1 \leqslant \beta<k$. Then by integration by parts, we have

$$
\begin{aligned}
\int_{0}^{a} t^{-\alpha}(1-\log t)^{\beta} d t= & c_{\alpha \beta k} \int_{0}^{a} t^{-\alpha}(1-\log t)^{\beta-k} d t+a^{1-\alpha} \sum_{j=0}^{k-1} c_{\alpha \beta j}(1-\log a)^{\beta-j} \\
& \quad\left(\text { using the fact that the function } t \rightarrow(1-\log t)^{\beta-k} \text { is increasing }\right) . \\
\leqslant & c_{\alpha \beta k}(1-\log a)^{\beta-k} \int_{0}^{a} t^{-\alpha} d t+c_{\alpha \beta k} a^{1-\alpha}(1-\log a)^{\beta} \\
\leqslant & c_{\alpha \beta} a^{1-\alpha}(1-\log a)^{\beta}
\end{aligned}
$$

If $\beta<0$ the inequality is still true since the function $t \rightarrow(1-\log t)^{\beta}$ is increasing, we argue as in the last line of the above proof. If $\beta \geqslant 0$ then $t \rightarrow(1-\log t)^{\beta}$ is decreasing, then the last result follows directly.

Using an argument of [3], we have:

\section{Proposition 2.3.}

For any $\alpha<-1, \beta \in \mathbb{R}$, we have

$$
\int_{a}^{1} t^{\alpha}(1-\log t)^{\beta} d t \lesssim a^{\alpha+1}(1-\log a)^{\beta}
$$

\section{Proof:}

Let $\varepsilon>0$ such that $\alpha+\varepsilon<-1$. For $N=1+\frac{|\beta|}{\varepsilon}, t^{-\varepsilon}(N-\log t)^{\beta}$ is decreasing. So $\int_{a}^{1} t^{\alpha}(1-\log t)^{\beta} d t \lesssim a^{-\varepsilon}(N-\log a)^{\beta} \int_{a}^{1} t^{\alpha+\varepsilon} d t \lesssim(1-\log a)^{\beta} a^{\alpha+1}$.

For convenience, we recall some of those Hardy type inequalities (see [3] Theorem 6.4, [18] Lemma 2.7, Corollary 2.9) that we will use. 


\section{Theorem 2.1.}

Suppose $\lambda>0,1 \leqslant b \leqslant+\infty$, and $-\infty<\beta<+\infty$, $\Phi$ a nonnegative measurable function on $(0,1)$. Then

$$
\int_{0}^{1}\left[t^{-\lambda}(1-\log t)^{\beta} \int_{0}^{t} \Phi(s) d s\right]^{b} \frac{d t}{t} \leqslant c \int_{0}^{1}\left[t^{1-\lambda}(1-\log t)^{\beta} \Phi(t)\right]^{b} \frac{d t}{t},
$$

and

$$
\int_{0}^{1}\left[t^{\lambda}(1-\log t)^{\beta} \int_{t}^{1} \Phi(s) d s\right]^{b} \frac{d t}{t} \leqslant c \int_{0}^{1}\left[t^{1+\lambda}(1-\log t)^{\beta} \Phi(t)\right]^{b} \frac{d t}{t} .
$$

The constant $c$ is independent of $\Phi$.

If $\Phi(t)=t^{\mu-1} \Phi_{1}(t), \mu>0, \Phi_{1}$ decreasing, then the above inequalities hold true for $0<b<1$.

In the above formula when $b=+\infty$, the integral is replaced by the supremum. In the case where $\lambda=0$ we use the following Hardy inequalities given in Bennett-Rudnick ([3] Theorem 6.5)

\section{Theorem 2.2.}

Suppose $1 \leqslant a \leqslant \infty, \alpha \in \mathbb{R}$ and $\alpha+\frac{1}{a} \neq 0$. Let $\psi$ be a nonnegative measurable function on $(0,1)$. Then, if $\alpha+\frac{1}{a}>0$,

$$
\left(\int_{0}^{1}\left[(1-\log t)^{\alpha} \int_{0}^{t} \psi(s) d s\right]^{a} \frac{d t}{t}\right]^{\frac{1}{a}} \leqslant c\left(\int_{0}^{1}\left[t(1-\log t)^{1+\alpha} \psi(t)\right]^{a} \frac{d t}{t}\right]^{\frac{1}{a}}
$$

and $\alpha+\frac{1}{a}<0$,

$$
\left(\int_{0}^{1}\left[(1-\log t)^{\alpha} \int_{t}^{1} \psi(s) d s\right]^{a} \frac{d t}{t}\right)^{\frac{1}{a}} \leqslant c\left(\int_{0}^{1}\left[t(1-\log t)^{1+\alpha} \psi(t)\right]^{a} \frac{d t}{t}\right)^{\frac{1}{a}} .
$$

The constant $c$ is independent of $\psi$.

Let $(\mathcal{R}, \mu)$ be a measure space and $\mathcal{M}(\mathcal{R}, \mu)$ be the set of all $\mu$ measurable functions over $\mathcal{R}$. A Banach space $X=X(\mathcal{R}, \mu)$ of $\mu$-measurable complex-valued functions in $\mathcal{M}(\mathcal{R}, \mu)$ ( set of all $\mu$ measurable functions over $\mathcal{R}$ ), equipped with the norm $\|\cdot\|_{X}$, is said to be a rearrangement-invariant Banach function space (shortly r.i. space) over $(\mathcal{R}, \mu)$ (or over $\mathcal{R}$ with respect to $\mu$ ) if the following five axioms hold:

$$
\begin{aligned}
& 0 \leq g \leq f \mu \text {-a.e. implies }\|g\|_{X} \leq\|f\|_{X} ; \\
& 0 \leq f_{n} \nearrow f \mu \text {-a.e. implies }\left\|f_{n}\right\|_{X} \nearrow\|f\|_{X} ;
\end{aligned}
$$




$$
\left\|\chi_{E}\right\|_{X}<\infty \text { for every } E \subset \mathcal{R} \text { of finite measure; }
$$

(P4) for every $E \subset \mathcal{R}$ with $\mu(E)<\infty$ there exists a constant $C_{E}$ such that $\int_{E}|f(x)| d \mu(x) \leq C_{E}\|f\|_{X}$ for every $f \in X$;

$$
\|f\|_{X}=\|g\|_{X} \text { whenever } f_{*}=g_{*} .
$$

Given an r.i. space $X$ on $(\mathcal{R}, \mu)$, the set

$$
X^{\prime}=\left\{f \in \mathcal{M}(\mathcal{R}, \mu): \int_{\mathcal{R}}|f(x) g(x)| d \mu(x)<\infty \text { for every } g \in X\right\},
$$

equipped with the norm

$$
\|f\|_{X^{\prime}}=\sup _{\|g\|_{X} \leq 1} \int_{\mathcal{R}}|f(x) g(x)| d \mu(x)=\sup _{\|g\|_{X} \leq 1} \int_{0}^{\mu(\mathcal{R})} f_{*}(t) g_{*}(t) d t,
$$

is called the associate space of $X$. It turns out that $X^{\prime}$ is again an r.i. space over $\mathcal{R}$ with respect to $\mu$ and that $X^{\prime \prime}=X$.

For every r.i. space $X$ over $(\mathcal{R}, \mu)$, there exists a unique r.i. space $\bar{X}$ over $(0, \infty)$ with respect to the one-dimensional Lebesgue measure, satisfying

$$
\|f\|_{X}=\left\|f_{*}\right\|_{\bar{X}}
$$

for every $f \in X$. This space, equipped with the norm

$$
\|f\|_{\bar{X}}=\sup _{\|g\|_{X^{\prime}} \leq 1} \int_{0}^{\mu(\mathcal{R})} f_{*}(t) g_{*}(t) d t
$$

is called the representation space of $X$.

The fundamental function of a r.i. Banach space, $X$, is defined by

$$
\Phi_{X}(t)=\left\|\chi_{[0, t]}\right\|_{X}, \quad t \in(0, \mu(\mathcal{R})) .
$$

. There is no loss of generality if we assume $\Phi_{X}$ to be positive, nondecreasing, absolutely continuous far from the origin, concave and to satisfy

$$
\Phi_{X}(t) \Phi_{X^{\prime}}(t)=t \quad \text { for all } \quad t \in(0, \mu(\mathcal{R})) .
$$

Details and further material on r.i. spaces can be found in [5, Chapter 2].

Note that (see [8]) grand Lebesgue spaces $L^{p), \alpha}(\Omega)$ and small Lebesgue spaces $L^{(p, \alpha}(\Omega)$ are r.i. spaces over $\Omega$ and

$$
\Phi_{L^{p), \alpha}}(t) \approx t^{\frac{1}{p}}(1-\log t)^{\frac{-\alpha}{p}} \text { and } \Phi_{L^{(p, \alpha}}(t) \approx t^{\frac{1}{p}}(1-\log t)^{\frac{\alpha}{p^{\prime}}}
$$

$\left(L^{p), \alpha}\right)^{\prime}=L^{\left(p^{\prime}, \alpha\right.}$.

Let $A_{0}$ and $A_{1}$ be two r.i. Banach spaces over $[0,1]$ and let $\Phi_{0}, \Phi_{1}$ be respectively their fundamental functions. We suppose that the following conditions are satisfied: 
i) There exists a constant $\mathrm{C}$ such that, for $i=0 ; 1$ and for all $t>0$

$$
\int_{0}^{t} \frac{d s}{\Phi_{i}(s)} \leq \frac{C t}{\Phi_{i}(t)}
$$

ii) There exists a constant $\mathrm{C}$ such that, for all $t>0$

$$
\begin{aligned}
& \frac{\Phi_{1}(t)}{\Phi_{0}(t)}\left\|\frac{\chi_{[0, t]}}{\Phi_{1}}\right\|_{A_{0}} \leq C, \\
& \frac{\Phi_{0}(t)}{\Phi_{1}(t)}\left\|\frac{\chi_{[t, 1]}}{\Phi_{0}}\right\|_{A_{1}} \leq C .
\end{aligned}
$$

Lemma 2.1. [4, Lemma 2.2] Let $A_{0}$ and $A_{1}$ be two r.i. Banach function spaces satisfied (C.0), (C.1) and (C.2) Then

$$
K\left(f, \frac{\Phi_{0}(t)}{\Phi_{1}(t)} ; A_{0}, A_{1}\right) \approx\left\|f_{*} \chi_{[0, t]}\right\|_{A_{0}}+\frac{\Phi_{0}(t)}{\Phi_{1}(t)}\left\|f_{*} \chi_{[t, 1]}\right\|_{A_{1}}, \quad 0<t<1 .
$$

\section{Remarks on the choice of the method}

We have chosen a direct method for the proof of our results by computing the $K$ functionals. In some part of the manuscript (for instance Theorem 5.1) we can adopt an alternative proof as using limiting reiteration theorems [1, 10, 11, 13]. Although, we observe that it is not possible to get the result without computations. The feature of our method is that, as a byproduct, we make explicit the behavior of the $K$-functional.

\section{Computation of some $K$-functionals and characterization of the interpolation spaces $\left(L^{p), \alpha}, L^{q), \alpha}\right)_{\theta, r}$}

We shall need few lemmas before reaching the proof of Theorem 1.1 and Theorem 1.2 .

\section{Lemma 3.1.}

Let $\sigma=\frac{p}{p-1}, 1<p<+\infty, \alpha>0$. Then

$$
\sup _{0<t<1} t^{-1}(1-\log t)^{-\frac{\alpha}{p}} \int_{0}^{t^{\sigma}} f_{*}(s) d s \leqslant c_{\alpha p} \sup _{0<t<1}(1-\log t)^{-\frac{\alpha}{p}}\left(\int_{t}^{1} f_{*}^{p}(s) d s\right)^{\frac{1}{p}}
$$

$0<t<1$, for some constant $c_{\alpha p}>0$. 


\section{Proof:}

We have

$$
\begin{aligned}
N_{1} \equiv & \sup _{0<t<1} t^{-1}(1-\log t)^{-\frac{\alpha}{p}} \int_{0}^{t^{\sigma}} f_{*}(s) d s \\
= & \sup _{0<t<1} t^{-1}(1-\log t)^{-\frac{\alpha}{p}} \int_{0}^{t^{\sigma}}\left(\frac{2}{s}\right)^{\frac{1}{p}}\left(\int_{\frac{s}{2}}^{s} d \tau\right)^{\frac{1}{p}} f_{*}(s) d s \\
\lesssim & \sup _{0<t<1} t^{-1}(1-\log t)^{-\frac{\alpha}{p}} \int_{0}^{t^{\sigma}} s^{-\frac{1}{p}}\left(\int_{s / 2}^{s} f_{*}^{p}(\tau) d \tau\right)^{\frac{1}{p}} d s \\
\lesssim & \sup _{0<t<1} t^{-1}(1-\log t)^{-\frac{\alpha}{p}}\left(\int_{0}^{t^{\sigma}} s^{-\frac{1}{p}}\left(1-\log \frac{s}{2}\right)^{\frac{\alpha}{p}} d s\right) \\
& \quad \times \sup _{0<s<t}\left(1-\log \frac{s}{2}\right)^{-\frac{\alpha}{p}}\left(\int_{s / 2}^{1} f_{*}^{p}(\tau) d \tau\right)^{\frac{1}{p}}
\end{aligned}
$$

Applying Proposition 2.2, we have

$$
\int_{0}^{t^{\sigma}} s^{-\frac{1}{p}}\left(1-\log \frac{s}{2}\right)^{\frac{\alpha}{p}} d s \leqslant c_{\alpha p} t^{\sigma\left(1-\frac{1}{p}\right)}(1-\log t)^{\frac{\alpha}{p}}
$$

From the two last inequalities, we have:

$$
N_{1} \leqslant c_{\alpha p} \sup _{0<t<1}(1-\log t)^{-\frac{\alpha}{p}}\left(\int_{t}^{1} f_{*}^{p}\right)^{\frac{1}{p}} \approx\|f\|_{p), \alpha} .
$$

The next lemma has been already proved (see Theorem 4.1 [15]) for a characterization of grand Lebesgue spaces as interpolation spaces between Lebesgue spaces, obtained using the Holmstedt's formula as well.

\section{Lemma 3.2.}

For $1<p<+\infty, \alpha>0$ we have

$$
L^{p), \alpha}=\left(L^{1}, L^{p}\right)_{1, \infty ;-\frac{\alpha}{p}}
$$

\section{Proof:}

According to the Holmstedt's formula (see [5]), we have for all $f \in L^{1}+L^{p}$, and for all $t \in(0,1)$

$$
K\left(f, t ; L^{1}, L^{p}\right) \approx \int_{0}^{t^{\sigma}} f_{*}(s) d s+t\left(\int_{t^{\sigma}}^{1} f_{*}^{p}(s) d s\right)^{\frac{1}{p}} \doteq K_{1 p}(f, t) \quad \text { with } \sigma=\frac{p}{p-1} .
$$


Making use of the definition of the norm in $\left(L^{1}, L^{p}\right)_{1, \infty ;-\frac{\alpha}{p}}$

$$
\begin{gathered}
\|f\|_{1, \infty ;-\frac{\alpha}{p}}=\sup _{0<t<1} t^{-1}(1-\log t)^{-\frac{\alpha}{p}} K_{1 p}(f, t) \\
\approx \sup _{0<t<1} t^{-1}(1-\log t)^{-\frac{\alpha}{p}} \int_{0}^{t^{\sigma}} f_{*}(s) d s+\sup _{0<t<1}(1-\log t)^{-\frac{\alpha}{p}}\left(\int_{t^{\sigma}}^{1} f_{*}^{p}(s) d s\right)^{\frac{1}{p}} .
\end{gathered}
$$

From Lemma 3.1, we deduce that

$$
\|f\|_{1, \infty ;-\frac{\alpha}{p}} \approx \sup _{0<t<1}(1-\log t)^{-\frac{\alpha}{p}}\left(\int_{t}^{1} f_{*}^{p}(s) d s\right)^{\frac{1}{p}}=\|f\|_{p), \alpha} .
$$

Noticing $1-\log t \approx 1-\log t^{\frac{1}{\sigma}}$.

\section{Lemma 3.3.}

Let $1<p<q, \alpha>0$. Then

$$
\sup _{0<t<1} t^{\frac{1}{q}-\frac{1}{p}}(1-\log t)^{-\frac{\alpha}{q}}\left(\int_{0}^{t} f_{*}^{p}(s) d s\right)^{\frac{1}{p}} \leqslant c_{\alpha p q} \sup _{0<t<1}(1-\log t)^{-\frac{\alpha}{q}}\left(\int_{t}^{1} f_{*}^{q}(s) d s\right)^{\frac{1}{q}}
$$

for some constant $c_{\alpha p q}>0$.

\section{Proof:}

The relation (8) is equivalent to

$$
\sup _{0<t<1} t^{-1}(1-\log t)^{-\frac{\alpha p}{q}}\left(\int_{0}^{t^{\sigma}} f_{*}(s) d s\right) \lesssim \sup _{0<t<1}(1-\log t)^{-\frac{\alpha p}{q}}\left(\int_{t}^{1} f_{*}(s)^{\frac{q}{p}} d s\right)^{\frac{p}{q}}
$$

with $\sigma=\frac{r}{r-1}, r=\frac{q}{p}>1$.

So we deduce Lemma 3.3 from Lemma 3.1, replacing $p$ by $r$.

\section{Theorem 3.1.}

Let $1 \leqslant p<q, \alpha>0$. Then

$$
L^{q), \alpha}(\Omega)=\left(L^{p}(\Omega), L^{q}(\Omega)\right)_{1, \infty ;-\frac{\alpha}{q}} .
$$

\section{Proof of Theorem 3.1}

According to Holmstedt's formula [5] we have for all $f \in L^{p}+L^{q}$, all $t \in(0,1)$

$$
K\left(f, t ; L^{p}, L^{q}\right) \approx\left(\int_{0}^{t^{\sigma}} f_{*}^{p}(s) d s\right)^{\frac{1}{p}}+t\left(\int_{t^{\sigma}}^{1} f_{*}^{q}(s) d s\right)^{\frac{1}{q}} \doteq K_{p q}(f, t)
$$


with $\sigma=\frac{p q}{q-p}$.

According to the norm of $f$ in $\left(L^{p}, L^{q}\right)_{1, \infty ;-\frac{\alpha}{q}}$ we have:

$$
\begin{aligned}
\|f\|_{1, \infty ;-\frac{\alpha}{q}} & \approx \sup _{0<t<1} t^{-1}(1-\log t)^{-\frac{\alpha}{q}} K_{p q}(f, t) \\
& \approx \sup _{0<t<1} t^{-1}(1-\log t)^{-\frac{\alpha}{q}}\left(\int_{0}^{t^{\sigma}} f_{*}^{p}(s) d s\right)^{\frac{1}{p}}+\sup _{0<t<1}(1-\log t)^{-\frac{\alpha}{q}}\left(\int_{t^{\sigma}}^{1} f_{*}^{q}(s) d s\right)^{\frac{1}{q}} \\
& =I_{1}+I_{2} .
\end{aligned}
$$

The first term $I_{1}$ of relation (10) is equivalent to

$$
\sup _{0<\tau<1} \tau^{\frac{1}{q}-\frac{1}{p}}(1-\log \tau)^{-\frac{\alpha}{q}}\left(\int_{0}^{\tau} f_{*}^{p}(s) d s\right)^{\frac{1}{p}} .
$$

(making use of the change of variables $\tau=t^{\sigma}$ and knowing as for $I_{2}, 1-\log \tau^{\frac{1}{\sigma}} \approx$ $1-\log \tau)$.

According Lemma 3.3, this last term is dominated by $I_{2}$.

Therefore we have

$$
\|f\|_{1, \infty ;-\frac{\alpha}{q}} \approx I_{1}+I_{2} \approx I_{2}=\|f\|_{q), \alpha} .
$$

Theorem 3.2. (Computation of the $K$-functional for the couple $\left(L^{p), \alpha}, L^{q}\right)$ ) Let $1<p<q, \alpha>0$. Then

$$
\begin{aligned}
K\left(f, t ; L^{p), \alpha}, L^{q}\right) & \approx \sup _{0<s<\varphi(t)}(1-\log s)^{-\frac{\alpha}{p}}\left(\int_{s}^{\varphi(t)} f_{*}^{p}(x) d x\right)^{\frac{1}{p}}+t\left(\int_{\varphi(t)}^{1} f_{*}^{q}(s) d s\right)^{\frac{1}{q}} \\
& =\bar{K}_{p q}(f, t)
\end{aligned}
$$

where $\varphi$ is the inverse of the increasing function $\psi(t)=t^{\frac{1}{p}-\frac{1}{q}}(1-\log t)^{-\frac{\alpha}{p}}, \quad t \in(0,1)$. Thus

$$
t=\varphi(t)^{\frac{1}{p}-\frac{1}{q}}(1-\log \varphi(t))^{-\frac{\alpha}{p}}
$$

\section{Proof of Theorem 3.2}

To apply Lemma 2.1, we need to check that the conditions (C.0), (C.1) and (C.2) are satisfied. Let $\Phi_{0}(t)=\left\|\chi_{[0, t]}\right\|_{L^{p), \alpha}}$ and $\Phi_{1}(t)=\left\|\chi_{[0, t]}\right\|_{L^{q}}$. As we have

$$
\Phi_{0}(t) \approx t^{\frac{1}{p}}(1-\log t)^{-\frac{\alpha}{p}}, \quad \Phi_{1}(t) \approx t^{\frac{1}{q}},
$$


we have that $\psi(t)=\frac{\Phi_{0}(t)}{\Phi_{1}(t)}$. The conditions (C.0) (C.1) and (C.2) easily follow by using the Proposition 2.2 and Proposition 2.3, and using Lemma 2.1 we get desired result. By same way we can obtain following theorem

Theorem 3.3. (Computation of the $K$-functional for the couple $\left(L^{p), \alpha}, L^{q), \alpha}\right)$ ) Let $1<p<q, \alpha>0$. Then

$$
\begin{aligned}
K\left(f, t ; L^{p), \alpha}, L^{q), \alpha}\right) & \approx \sup _{0<s<\varphi(t)}(1-\log s)^{-\frac{\alpha}{p}}\left(\int_{s}^{\varphi(t)} f_{*}^{p}(x) d x\right)^{\frac{1}{p}} \\
& +t \sup _{\varphi(t)<s<1}(1-\log s)^{-\frac{\alpha}{q}}\left(\int_{s}^{1} f_{*}^{q}(x) d x\right)^{\frac{1}{q}},
\end{aligned}
$$

where $\varphi$ is the inverse of the increasing function $\psi(t)=t^{\frac{1}{p}-\frac{1}{q}}(1-\log t)^{-\frac{\alpha}{p}+\frac{\alpha}{q}}, \quad t \in(0,1)$. Thus

$$
t=\varphi(t)^{\frac{1}{p}-\frac{1}{q}}(1-\log \varphi(t))^{-\frac{\alpha}{p}+\frac{\alpha}{q}}
$$

\section{Proof of Theorem 1.1}

Let $f$ be in $\left(L^{p), \alpha}, L^{q}\right)_{1, \infty ;-\frac{\alpha}{q}}$. Then, its norm is

$$
\|f\|_{1, \infty ;-\frac{\alpha}{q}}=\sup _{0<t<1} t^{-1}(1-\log t)^{\frac{-\alpha}{q}} K\left(f, t ; L^{p), \alpha}, L^{q}\right) .
$$

Following Theorem 3.2 , this expression gives:

$$
\begin{aligned}
\|f\|_{1, \infty ;-\frac{\alpha}{q}} \approx & \sup _{0<t<1} t^{-1}(1-\log t)^{\frac{-\alpha}{q}} \sup _{0<s<\varphi(t)}(1-\log s)^{\frac{-\alpha}{p}}\left(\int_{s}^{\varphi(t)} f_{*}^{p}(\tau) d \tau\right)^{\frac{1}{p}} \\
& +\sup _{0<t<1}(1-\log t)^{\frac{-\alpha}{q}}\left(\int_{\varphi(t)}^{1} f_{*}^{q}(\tau) d \tau\right)^{\frac{1}{q}}=J_{1}+J_{2} .
\end{aligned}
$$

But we have by the definition of $\varphi$,

$$
t^{-1}=\varphi(t)^{\frac{1}{q}-\frac{1}{p}}(1+|\log \varphi(t)|)^{\frac{\alpha}{p}}, \quad 1+|\log \varphi| \approx 1+|\log t| .
$$

Thus the first term $J_{1}$ of (11), after a change of variables, gives

$$
J_{1} \approx \sup _{0<t<1} t^{\frac{p-q}{q p}}(1-\log t)^{\frac{\alpha(q-p)}{p q}} \sup _{0<s<t}(1-\log s)^{\frac{-\alpha}{p}}\left(\int_{s}^{t} f_{*}^{p}(\tau) d \tau\right)^{\frac{1}{p}} .
$$

But we can bound the last term of (12) as:

$$
\sup _{0<s<t}(1-\log s)^{\frac{-\alpha}{p}}\left(\int_{s}^{t} f_{*}^{p}(\tau) d \tau\right)^{\frac{1}{p}} \leqslant t^{\frac{q-p}{p q}} \sup _{0<s<t}(1-\log s)^{\frac{\alpha(p-q)}{q p}} \sup _{0<s<t}(1-\log s)^{\frac{-\alpha}{q}}\left(\int_{s}^{t} f_{*}^{q}(\tau) d \tau\right)^{\frac{1}{q}}
$$


(using Hölder inequality and introducing new factor).

Therefore, we can estimate $J_{1}$ (after simplifying):

$$
J_{1} \lesssim \sup _{0<s<1}(1-\log s)^{\frac{-\alpha}{q}}\left(\int_{s}^{1} f_{*}^{q}(\tau) d \tau\right)^{\frac{1}{q}}=\|f\|_{q), \alpha} .
$$

While for the second term $J_{2}$, we have:

$$
J_{2} \approx \sup _{0<t<1}(1-\log \varphi(t))^{\frac{-\alpha}{q}}\left(\int_{\varphi(t)}^{1} f_{*}^{q}(\tau) d \tau\right)^{\frac{1}{q}}
$$

which implies

$$
J_{2} \approx \sup _{0<s<1}(1-\log s)^{\frac{-\alpha}{q}}\left(\int_{s}^{1} f_{*}^{q}(\tau) d \tau\right)^{\frac{1}{q}}=\|f\|_{q), \alpha} .
$$

We then have from relations (11) to (15)

$$
J_{2} \lesssim\|f\|_{1, \infty ;-\frac{\alpha}{q}} \approx J_{1}+J_{2}
$$

This shows the result.

Next, we want to prove Theorem 1.2 .

For this, we shall need the:

\section{Lemma 3.4.}

Let $1<p<q, \alpha>0,0<\theta<1,1 \leqslant r<+\infty$. Then

$$
\left(L^{p), \alpha}, L^{q), \alpha}\right)_{\theta, r}=\left(L^{p), \alpha}, L^{q}\right)_{\theta, r ;-\frac{\alpha \theta}{q}} \cdot
$$

\section{Proof:}

From Theorem 1.1, we know that

$$
\left(L^{p), \alpha}, L^{q), \alpha}\right)_{\theta, r}=\left(L^{p), \alpha},\left(L^{p), \alpha}, L^{q}\right)_{1, \infty ;-\frac{\alpha}{q}}\right)_{\theta, r} .
$$

In this case, we can apply the Holmstedt formula (see Theorem 3.1 in [18]) valid also for the extreme case $\theta_{1}=1$.

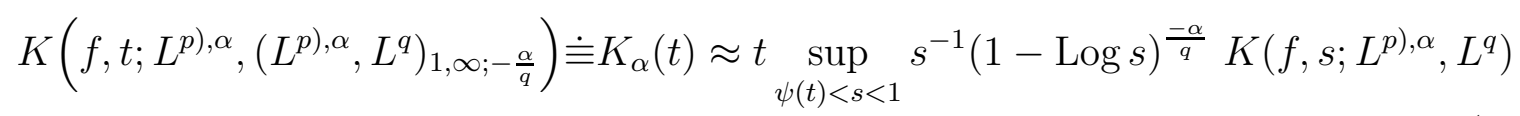

where $\psi$ is an invertible function from $[0,1]$ into itself according to Proposition 2.1 so that

$$
t \approx \psi(t)(1-\log \psi(t))^{\frac{\alpha}{q}} \quad \text { for } t \in[0,1]
$$


Considering the norm $\|f\|_{\theta, r}$ of $f$ in $\left(L^{p), \alpha}, L^{q), \alpha}\right)_{\theta, r}$ we have:

$$
\begin{aligned}
\|f\|_{\theta, r} & \approx\left\|t^{-\theta} K_{\alpha}(t)\right\|_{L^{r}\left(0,1 ; \frac{d t}{t}\right)} \\
& \approx\left\|t^{1-\theta} \sup _{\psi(t)<s<1} s^{-1}(1-\log s)^{\frac{-\alpha}{q}} K\left(f, s ; L^{p), \alpha}, L^{q}\right)\right\|_{L^{r}\left(0,1 ; \frac{d t}{t}\right)} \\
& \approx\left\|\psi(t)^{1-\theta}(1-\log \psi(t))^{\frac{\alpha(1-\theta)}{q}} \sup _{\psi(t)<s<1} s^{-1}(1-\log s)^{\frac{-\alpha}{q}} K\left(f, s ; L^{p), \alpha}, L^{q}\right)\right\|_{L^{r}\left(\frac{d t}{t}\right)} \\
& \approx\left\|t^{1-\theta}(1-\log t)^{\frac{\alpha(1-\theta)}{q}} \sup _{t<s<1} s^{-1}(1-\log s)^{\frac{-\alpha}{q}} K\left(f, s ; L^{p), \alpha}, L^{q}\right)\right\|_{L^{r}\left(\frac{d t}{t}\right)} .
\end{aligned}
$$

The last line is obtained after the change of variables $t \rightarrow \psi(t)$. To control this last term, we need

\section{Proposition 3.1.}

Let $K_{d}$ be a decreasing nonnegative function on $(0,1)$ and $p, q, \alpha, \theta, r$ are as in Lemma 3.4. Then

$$
\begin{gathered}
I_{r} \doteq \int_{0}^{1}\left[t^{1-\theta}(1-\log t)^{\frac{\alpha(1-\theta)}{q}} \sup _{t<s<1}(1-\log s)^{\frac{-\alpha}{q}} K_{d}(s)\right]^{r} \frac{d t}{t} \\
\approx \int_{0}^{1}\left[t^{1-\theta}(1-\log t)^{\frac{-\alpha \theta}{q}} K_{d}(t)\right]^{r} \frac{d t}{t} \doteq I_{d} .
\end{gathered}
$$

\section{Proof:}

Since $\sup _{t<s<1}(1-\log s)^{\frac{-\alpha}{q}} K_{d}(s) \geqslant(1-\log t)^{\frac{-\alpha}{q}} K_{d}(t)$, we deduce

$$
I_{r} \geqslant \int_{0}^{1}\left[t^{1-\theta}(1-\log t)^{\frac{-\alpha \theta}{q}} K_{d}(t)\right]^{r} \frac{d t}{t}=I_{d}
$$

For the reverse inequality, since $K_{d}$ is decreasing and $s \rightarrow(1-\log s)^{\frac{-\alpha r}{q}}$ increasing then,

$$
(1-\log s)^{\frac{-\alpha r}{q}} K_{d}^{r}(s) \lesssim \int_{s / 2}^{s}(1-\log x)^{\frac{-\alpha r}{q}} K_{d}^{r}(x) \frac{d x}{x} \lesssim\left(1-\log \frac{s}{2}\right)^{\frac{-\alpha r}{q}} K_{d}^{r}\left(\frac{s}{2}\right) .
$$

Setting

$$
I_{1 r}=\int_{0}^{1} t^{(1-\theta) r}(1-\log t)^{\frac{\alpha r(1-\theta)}{q}} \sup _{t<s<1}\left(\int_{s / 2}^{s}(1-\log x)^{\frac{-\alpha r}{q}} K_{d}^{r}(x) \frac{d x}{x}\right) \frac{d t}{t},
$$

thus, we can bound, the integral $I_{r}$ by $I_{1 r}$ :

$$
I_{r}=\int_{0}^{1} t^{(1-\theta) r}(1-\log t)^{\frac{\alpha r(1-\theta)}{q}} \sup _{t<s<1}(1-\log s)^{\frac{-\alpha r}{q}} K_{d}^{r}(s) \frac{d t}{t} \lesssim I_{1 r}
$$


and

$$
I_{1 r} \lesssim \int_{0}^{1} t^{(1-\theta) r}(1-\log t)^{\frac{\alpha r(1-\theta}{q}} \sup _{t<s<1}\left(1-\log \frac{s}{2}\right)^{\frac{-\alpha r}{q}} K_{d}^{r}\left(\frac{s}{2}\right) \frac{d t}{t}=I_{2 r} .
$$

By a change of variables in the term $I_{2 r}$, we have

$$
I_{2 r} \lesssim I_{r}
$$

Therefore, relations (19) and (20) imply:

$$
I_{r} \approx I_{1 r}
$$

We may bound $I_{1 r}$ as:

$$
I_{1 r} \leqslant \int_{0}^{1} t^{(1-\theta) r}(1-\log t)^{\frac{\alpha r(1-\theta)}{q}}\left(\int_{t / 2}^{1}(1-\log x)^{\frac{-\alpha r}{q}} K_{d}^{r}(x) \frac{d x}{x}\right) \frac{d t}{t} .
$$

By Fubini's Theorem, the upperbound in this inequality gives:

$$
I_{1 r} \lesssim \int_{0}^{1}(1-\log x)^{\frac{-\alpha r}{q}} K_{d}^{r}(x)\left(\int_{0}^{2 x} t^{(1-\theta) r}(1-\log t)^{\frac{\alpha r(1-\theta)}{q}} \frac{d t}{t}\right) \frac{d x}{x} .
$$

From Proposition 2.2, we have

$$
\int_{0}^{2 x} t^{(1-\theta) r-1}(1-\log t)^{\frac{\alpha r(1-\theta)}{q}} d t \leqslant c_{0 q \alpha} x^{(1-\theta) r}(1-\log x)^{\frac{\alpha r(1-\theta)}{q}} .
$$

From relation (21), the last estimate $I_{1 r}$ becomes

$$
I_{1 r} \lesssim \int_{0}^{1} x^{(1-\theta) r}(1-\log x)^{\frac{-\alpha r \theta}{q}} K_{d}^{r}(x) \frac{d x}{x}=I_{d} .
$$

With relation (19) we get:

$$
I_{r} \lesssim I_{1 r} \lesssim I_{d} \leqslant I_{r}
$$

\section{End of proof of Lemma 3.4}

We apply Proposition 3.1 with

$$
K_{d}(t)=t^{-1} K\left(f, t ; L^{p), \alpha}, L^{q}\right), t \in(0,1)
$$

to derive the:

$$
\begin{aligned}
\|f\|_{\theta, r} & \approx\left\|t^{1-\theta}(1-\log t)^{\frac{\alpha(1-\theta)}{q}} \sup _{t<s<1}(1-\log s)^{\frac{-\alpha}{q}} K_{d}(s)\right\|_{L^{r}\left(\frac{d t}{t}\right)} \\
& \approx I_{d}^{\frac{1}{r}}=\|f\|_{V} \text { with } V=\left(L^{p), \alpha}, L^{q}\right)_{\theta, r ;-\frac{\alpha \theta}{q}} .
\end{aligned}
$$




\section{Proof of Theorem 1.2}

Let

$$
V=\left(L^{p), \alpha}, L^{q), \alpha}\right)_{\theta, r} \quad \text { and } \quad L^{p_{\theta}, r}(\log L)^{-\frac{\alpha}{p_{\theta}}}=V_{\theta}
$$

with $\frac{1}{p_{\theta}}=\frac{1-\theta}{p}+\frac{\theta}{q}$.

We recall that, for $f \in V$

$$
\mid f \|_{V}^{r}=\int_{0}^{1}\left[t^{-\theta}(1-\log t)^{\frac{-\alpha \theta}{q}} K\left(f, t ; L^{p), \alpha}, L^{q), \alpha}\right)\right]^{r} \frac{d t}{t} .
$$

Applying Theorem 3.3, we have

$$
\begin{aligned}
\|f\|_{V}^{r} \approx & \int_{0}^{1}\left[t^{-\theta} \sup _{0<s<\varphi(t)}(1-\log s)^{\frac{-\alpha}{p}}\left(\int_{s}^{\varphi(t)} f_{*}^{p}(\tau) d \tau\right)^{\frac{1}{p}}\right]^{r} \frac{d t}{t} \\
& +\int_{0}^{1}\left[t^{1-\theta} \sup _{\varphi(t)<s<1}(1-\log s)^{\frac{-\alpha}{q}}\left(\int_{s}^{1} f_{*}^{q}(\tau) d \tau\right)^{\frac{1}{q}}\right]^{r} \frac{d t}{t} \\
= & I I_{1}+I I_{2} .
\end{aligned}
$$

From the first term $I I_{1}$, we know that

$$
t=\varphi(t)^{\frac{1}{p}-\frac{1}{q}}(1-\log \varphi(t))^{\frac{-\alpha}{p}+\frac{\alpha}{q}}
$$

and

$$
1-\log t \approx 1-\log \varphi(t)
$$

Therefore, we have

$$
t^{-\theta} \approx \varphi(t)^{\theta\left(\frac{1}{q}-\frac{1}{p}\right)}(1-\log \varphi(t))^{\frac{-\alpha \theta(p-q)}{q p}} .
$$

Replacing the integrand in the integral by the $I I_{1}$ of (23) and making the change of variables $x=\varphi(t)$ or equivalently $t=\psi(x)$, we have

$$
I I_{1} \approx \int_{0}^{1}\left[x^{\theta\left(\frac{1}{q}-\frac{1}{p}\right)}(1-\log x)^{\frac{-\alpha \theta(p-q)}{q p}} \sup _{0<s<x}(1-\log s)^{\frac{-\alpha}{p}}\left(\int_{s}^{x} f_{*}^{p}(\tau) d \tau\right)^{\frac{1}{p}}\right]^{r} \frac{d x}{x} .
$$

Thus the monotony of $f_{*}$ leads to:

$$
I I_{1} \gtrsim \int_{0}^{1}\left[x^{\theta\left(\frac{1}{q}-\frac{1}{p}\right)}(1-\log x)^{\frac{-\alpha \theta(p-q)}{q p}}\left(1-\log \frac{x}{2}\right)^{\frac{-\alpha}{p}} x^{\frac{1}{p}} f_{*}(x)\right]^{r} \frac{d x}{x}
$$




$$
I I_{1} \gtrsim \int_{0}^{1}\left[t^{\frac{1-\theta}{p}+\frac{\theta}{q}}(1-\log t)^{\frac{-\alpha(q(1-\theta)+\theta p)}{p q}} f_{*}(t)\right]^{r} \frac{d t}{t}=\|f\|_{V_{\theta}}^{r} .
$$

In particular, we have shown that

$$
\|f\|_{V} \gtrsim\|f\|_{V_{\theta}} \text {. }
$$

For the reverse of relation (25), let us consider $\varepsilon>0$ small enough so that

$$
\begin{gathered}
\theta\left(\frac{1}{q}-\frac{1}{p}\right)+\frac{\varepsilon}{p}<0 . \\
I I_{1} \lesssim \int_{0}^{1}\left[x^{\theta\left(\frac{1}{q}-\frac{1}{p}\right)}(1-\log x)^{\frac{-\alpha \theta(p-q)}{q p}} \sup _{0<s<x}(1-\log s)^{\frac{-\alpha}{p}}\left(\int_{s}^{x} t^{\varepsilon-1} t^{1-\varepsilon} f_{*}^{p}(t) d t\right)^{\frac{1}{p}}\right]^{r} \frac{d x}{x} .
\end{gathered}
$$

Then

$$
I I_{1} \lesssim \int_{0}^{1}\left[x^{\theta\left(\frac{1}{q}-\frac{1}{p}\right)+\frac{\varepsilon}{p}}(1-\log x)^{\frac{-\alpha}{p_{\theta}}} \sup _{0<t<x} t^{\frac{1-\varepsilon}{p}} f_{*}(t)\right]^{r} \frac{d x}{x} .
$$

We have the following

\section{Proposition 3.2.}

For all $x>0$

$$
\sup _{0<t<x} t^{\frac{1-\varepsilon}{p}} f_{*}(t) \leqslant 2(\log 2)^{\frac{1}{r^{\prime}}}\left(\int_{0}^{x} s^{r \frac{1-\varepsilon}{p}-1} f_{*}^{r}(s) d s\right)^{\frac{1}{r}},
$$

with $\frac{1}{r^{\prime}}+\frac{1}{r}=1$.

\section{Proof:}

For any $s \in\left[\frac{t}{2}, t\right]$, we have

$$
t^{\frac{1-\varepsilon}{p}-1} f_{*}(t) \leqslant s^{\frac{1-\varepsilon}{p}-1} f_{*}(s)
$$

since $\frac{1}{p}-1-\frac{\varepsilon}{p}<0$.

Integrating between $\frac{t}{2}$ and $t$, and using Hölder inequality, we have

$$
\begin{aligned}
& t^{\frac{1-\varepsilon}{p}} f_{*}(t) \leqslant 2\left(\int_{t / 2}^{t} s^{\frac{1-\varepsilon}{p} r-1} f_{*}^{r}(s) d s\right)^{\frac{1}{r}}(\log 2)^{\frac{1}{r^{\prime}}} \\
& t^{\frac{1-\varepsilon}{p}} f_{*}(t) \leqslant 2\left(\int_{0}^{t} s^{\frac{1-\varepsilon}{p} r-1} f_{*}^{r}(s) d s\right)^{\frac{1}{r}}(\log 2)^{\frac{1}{r^{\prime}}} .
\end{aligned}
$$

From which, we derive the result. 
Using this Proposition 3.2 , setting $m=\theta\left(\frac{1}{q}-\frac{1}{p}\right)+\frac{\varepsilon}{p}<0$,

$$
I I_{1} \lesssim \int_{0}^{1} x^{m r-1}(1-\log x)^{\frac{-\alpha r}{p_{\theta}}}\left(\int_{0}^{x} s^{\frac{1-\varepsilon}{p} r-1} f_{*}^{r}(s) d s\right) d x .
$$

Applying Hardy type inequality (see Theorem 2.1 and [3], Theorem 6.4 ), relation (26) becomes

$$
I I_{1} \lesssim \int_{0}^{1}\left[s^{\frac{1}{p_{\theta}}}(1-\log s)^{\frac{-\alpha}{p_{\theta}}} f_{*}(s)\right]^{r} \frac{d s}{s}=\|f\|_{L^{p_{\theta}, r}(\log L)^{-\frac{\alpha}{p_{\theta}}}}^{r} .
$$

It remains to show that the second term satisfies:

$$
I I_{2}=\int_{0}^{1}\left[t^{1-\theta} \sup \varphi(t)<s<1(1-\log s)^{\frac{-\alpha \theta}{q}}\left(\int_{s}^{1} f_{*}^{q}(\tau) d \tau\right)^{\frac{1}{q}}\right]^{r} \frac{d t}{t} \lesssim\|f\|_{V_{\theta}}^{r} .
$$

For this, we recall that $\varphi$ is the inverse of the function $\psi(x)=x^{\frac{1}{p}-\frac{1}{q}}(1-\log x)^{\frac{-\alpha}{p}+\frac{\alpha}{q}}, \quad x \in$ $[0,1]$ so we have the following relations:

$$
\begin{gathered}
t=\varphi(t)^{\frac{1}{p}-\frac{1}{q}}(1-\log \varphi(t))^{\frac{-\alpha}{p}+\frac{\alpha}{q}}, \\
1-\log \varphi(t) \approx 1-\log t,
\end{gathered}
$$

and

$$
\frac{\psi^{\prime}(x)}{\psi(x)} \approx \frac{1}{x}
$$

Therefore, we rewrite $I I_{2}$ as:

$I I_{2} \approx \int_{0}^{1}\left[\varphi(t)^{(1-\theta)\left(\frac{1}{p}-\frac{1}{q}\right)}(1-\log \varphi(t))^{\frac{-\alpha(1-\theta)}{p}+\frac{\alpha(1-\theta)}{q}} \sup _{\varphi(t)<s<1}(1-\log s)^{\frac{-\alpha}{q}}\left(\int_{s}^{1} f_{*}^{q}(\tau) d \tau\right)^{\frac{1}{q}}\right]^{r} \frac{d t}{t}$.

Making the change of variables, $t=\psi(x) \Longleftrightarrow x=\varphi(t)$

$I I_{2} \approx \int_{0}^{1}\left[x^{(1-\theta)\left(\frac{1}{p}-\frac{1}{q}\right)}(1-\log x)^{\frac{-\alpha(1-\theta)}{p}+\frac{\alpha(1-\theta)}{q}} \sup _{x<t<1}(1-\log t)^{\frac{-\alpha}{q}}\left(\int_{t}^{1} f_{*}^{q}(s) d s\right)^{\frac{1}{q}}\right]^{r} \frac{d x}{x}$.

Applying Proposition 3.1, we have :

$$
\begin{gathered}
\int_{0}^{1}\left[x^{(1-\theta)\left(\frac{1}{p}-\frac{1}{q}\right)}(1-\right. \\
\left.\log x)^{\frac{-\alpha(1-\theta)}{p}+\frac{\alpha(1-\theta)}{q}} \sup _{x<t<1}(1-\log t)^{\frac{-\alpha}{q}}\left(\int_{t}^{1} f_{*}^{q}(s) d s\right)^{\frac{1}{q}}\right]^{r} \frac{d x}{x} \\
\leqslant c \int_{0}^{1}\left[x^{\frac{1}{p_{\theta}}}(1-\log x)^{\frac{-\alpha}{p_{\theta}}} f_{*}(x)\right]^{r} \frac{d x}{x} \equiv c\|f\|_{V_{\theta}}^{r} .
\end{gathered}
$$

From the preceding results, we can characterize the interpolation spaces for small Lebesgue spaces. 
Theorem 3.4.

Let $0<\theta<1,1<r<+\infty, 1<p<q, \alpha>0$. Then

$$
\left(L^{(p, \alpha}, L^{(q, \alpha}\right)_{\theta, r}=L^{p_{\theta}, r}(\log L)^{\frac{\alpha}{p_{\theta}^{\prime}}}
$$

with $\frac{1}{p_{\theta}}=\frac{1-\theta}{p}+\frac{\theta}{q}, \frac{1}{p_{\theta}}+\frac{1}{p_{\theta}^{\prime}}=1$.

\section{Proof:}

By the duality result on interpolation spaces, (see [7, 22]), we have,

$$
\left[\left(L^{(p, \alpha}, L^{(q, \alpha}\right)_{\theta, r}\right]^{\prime}=\left(L^{\left.p^{\prime}\right), \alpha}, L^{\left.q^{\prime}\right), \alpha}\right)_{\theta, r^{\prime}}=\left(L^{\left.q^{\prime}\right), \alpha}, L^{\left.p^{\prime}\right), \alpha}\right)_{1-\theta, r^{\prime}}
$$

and noticing $\left(L^{\left.p^{\prime}\right), \alpha}\right)^{\prime}=L^{(p, \alpha}, L^{\infty}(\Omega)$ is in $L^{(p, \alpha} \cap L^{(q, \alpha}$ and dense in each of these spaces. From Theorem [1.2, since $q^{\prime}<p^{\prime}$, we have

$$
\left(L^{\left.q^{\prime}\right), \alpha}, L^{\left.p^{\prime}\right), \alpha}\right)_{1-\theta, r^{\prime}}=L^{q_{1-\theta}^{\prime}, r^{\prime}}(\log L)^{-\frac{\alpha}{q_{1-\theta}^{\prime}}}
$$

with $\frac{1}{q_{1-\theta}^{\prime}}=\frac{\theta}{q^{\prime}}+\frac{1-\theta}{p^{\prime}}=1-\frac{1}{p_{\theta}}=\frac{1}{p_{\theta}^{\prime}}$.

Thus taking the associate space in the above equation (30) gives, taking into account (29),

$$
\left(L^{(p, \alpha}, L^{(q, \alpha}\right)_{\theta, r}=\left[\left(L^{(p, \alpha}, L^{(q, \alpha}\right)_{\theta, r}\right]^{\prime \prime}=L^{p_{\theta}, r}(\log L)^{\frac{\alpha}{p_{\theta}^{\prime}}}
$$

with $\frac{1}{p_{\theta}}=\frac{1-\theta}{p}+\frac{\theta}{q}, \frac{1}{p_{\theta}}+\frac{1}{p_{\theta}^{\prime}}=1$.

A main consequence of Theorem 1.2 and Theorem 3.4 is the

\section{Theorem 3.5.}

Let $1<a<+\infty, \beta \in \mathbb{R}, \beta \neq 0,1<r<+\infty$. Then the Lorentz-Zygmund space $L^{a, r}(\log L)^{\beta}$ is an interpolation space in the sense of Peetre of two Grand Lebesgue spaces if $\beta<0$ and of two small Lebesgue spaces if $\beta>0$.

\section{Remark 3.1.}

If $\beta=0$, it is already known that the Lorentz space $L^{a, r}(\Omega)$ is an interpolation space of two classical Lebesgue spaces.

\section{Small Lebesgue space as interpolation of usual Lebesgue spaces}

The next proposition has been already proved on [10], we drop its proofs : 
Proposition 4.1. [10]

Let $1<p<+\infty, \alpha>0$. Then

$$
L^{(p, \alpha}=\left(L^{p}, L^{\infty}\right)_{0,1 ;-\frac{\alpha}{p}+\alpha-1} .
$$

\section{Proposition 4.2.}

Let $1<p<q, \alpha>0$. Then

$$
L^{(p, \alpha}=\left(L^{p}, L^{q}\right)_{0,1 ;-1+\alpha-\frac{\alpha}{p}} .
$$

\section{Proof:}

It is similar to the above Proposition 4.1. Indeed, let set $W_{p q}$ the space on the RHS, then for $f \in W_{p q}$

$$
\|f\|_{W_{p q}}=\int_{0}^{1}(1-\log t)^{-1-\frac{\alpha}{p}+\alpha} K\left(f, t ; L^{p}, L^{q}\right) \frac{d t}{t} .
$$

By the Holmstedt formula, we have

$$
K\left(f, t ; L^{p}, L^{q}\right) \approx\left(\int_{0}^{t^{\sigma}} f_{*}^{p}(s) d s\right)^{\frac{1}{p}}+t\left(\int_{t^{\sigma}}^{1} f_{*}^{q}(s) d s\right)^{\frac{1}{q}} \text { with } \frac{1}{\sigma}=\frac{q-p}{p q} .
$$

Then, we deduce the equivalent expression of the norm:

$$
\|f\|_{W_{p q}} \approx\|f\|_{L^{(p, \alpha}}+\int_{0}^{1}(1-\log t)^{-1-\frac{\alpha}{p}+\alpha} t^{\frac{1}{\sigma}}\left(\int_{t}^{1} f_{*}^{q}(s) d s\right)^{\frac{1}{q}} \frac{d t}{t} .
$$

Let us show that the last term in relation (31) is less or equal to the norm of $f$ in $L^{(p, \alpha}$. Let us temporarily set $\beta=-1+\alpha-\frac{\alpha}{p}$, let $1<\varepsilon<\frac{q}{\sigma}+1$, then

$$
\begin{aligned}
O_{1} & =\int_{0}^{1}\left[(1-\log t)^{\beta q} t^{\frac{q}{\sigma}-\varepsilon+1} t^{\varepsilon-1} \int_{t}^{1} f_{*}^{q}(x) d x\right]^{\frac{1}{q}} \frac{d t}{t} \\
& \leqslant \int_{0}^{1}\left[(1-\log t)^{\beta q} t^{\frac{q}{\sigma}-\varepsilon+1} \int_{t}^{1} x^{\varepsilon-1} f_{*}^{q}(x) d x\right]^{\frac{1}{q}} \frac{d t}{t} .
\end{aligned}
$$

Applying Hardy inequality (see Theorem 2.1] and [3] Theorem 6.4), we obtain from the relation (32)

$$
O_{1} \lesssim \int_{0}^{1}\left[(1-\log t)^{\beta} t^{\frac{1}{\sigma}+\frac{1}{q}} f_{*}(t)\right] \frac{d t}{t} .
$$

But $\frac{1}{\sigma}+\frac{1}{q}=\frac{1}{p}$ then $t^{\frac{1}{\sigma}+\frac{1}{q}} f_{*}(t) \leqslant\left(\int_{0}^{t} f_{*}^{p}(x) d x\right)^{\frac{1}{p}}$. So we derive

$$
O_{1} \lesssim \int_{0}^{1}\left[(1-\log t)^{\beta}\left(\int_{0}^{t} f_{*}^{p}(x) d x\right)^{\frac{1}{p}}\right] \frac{d t}{t}=\|f\|_{L^{(p, \alpha}} .
$$


Thus, we have from relations (31) to (34):

$$
\|f\|_{L^{(p, \alpha}} \lesssim\|f\|_{W_{p q}} \lesssim\|f\|_{L^{(p, \alpha}} .
$$

\section{Interpolation between small, Grand Lebesgue spaces and the associated $K$-functional}

In this section, we want to determine the interpolation space $\left(L^{(p, \alpha}, L^{q), \beta}\right)_{\theta, r}$. Due to the technical aspect of the proof, we shall only consider the case where $\alpha=\beta=1$, the argument remains the same in the general case.

We want to show the following theorem:

\section{Theorem 5.1.}

Let $0<\theta<1,1 \leqslant r<+\infty, p<q$. Then

$$
\left(L^{(p}, L^{q)}\right)_{\theta, r}=L^{p_{\theta}, r}(\log L)^{\alpha_{\theta}}
$$

where $\frac{1}{p_{\theta}}=\frac{1-\theta}{p}+\frac{\theta}{q}, \quad \alpha_{\theta}=1-\theta-\frac{1}{p_{\theta}}$.

We shall need the following $K$-functional.

Theorem 5.2.

Let $1<p<q<+\infty$. Then for all $t>0, f \in L^{(p}+L^{q)}$

$$
\begin{aligned}
K\left(f, t ; L^{(p}, L^{q)}\right) \approx & \int_{0}^{\varphi(t)}(1-\log s)^{\frac{-1}{p}}\left(\int_{0}^{s} f_{*}^{p}(\tau) d \tau\right)^{\frac{1}{p}} \frac{d s}{s}+(1-\log t)^{\frac{p-1}{p}}\left(\int_{0}^{\varphi(t)} f_{*}^{p}(\tau) d \tau\right)^{\frac{1}{p}} \\
& +t \sup _{\varphi(t)<s<1}(1-\log s)^{\frac{-1}{q}}\left(\int_{s}^{1} f_{*}^{q}(\tau) d \tau\right)^{\frac{1}{q}} \\
\doteq & \sum_{i=1}^{3} K_{i}(t)
\end{aligned}
$$

where $\varphi$ is an invertible function from [0,1] into itself satisfying the equivalence

$$
\varphi(t)^{\frac{1}{p}-\frac{1}{q}}(1-\log \varphi(t))^{\frac{p-q+p q}{p q}} \approx t .
$$




\section{Proof:}

We can apply Lemma 2.1 for $A_{0}=L^{(p}$ and $A_{1}=L^{q)}$. Let $\Phi_{0}(t)=\left\|\chi_{[0, t]}\right\|_{L^{(p p}}$ and $\Phi_{1}(t)=\left\|\chi_{[0, t]}\right\|_{L^{q}}$. As we have

$$
\Phi_{0}(t) \approx t^{\frac{1}{p}}(1-\log t)^{\frac{1}{p^{\prime}}}, \quad \Phi_{1}(t) \approx t^{\frac{1}{q}}(1-\log t)^{-\frac{1}{q}},
$$

we have that $\psi(t)=\frac{\Phi_{0}(t)}{\Phi_{1}(t)}$. As $p<q$ the conditions (C.0) (C.1) and (C.2) easily follow by using the Proposition 2.2 and Proposition 2.3. and from Lemma 2.1 we obtain the result.

Next, we want to prove Theorem 5.1. We need to show that the norm of $f$ in $W \doteq\left(L^{(p}, L^{q)}\right)_{\theta, r}$ is equivalent to its norm in $V=L^{p_{\theta}, r}(\log L)^{\alpha_{\theta}}$.

For commodity, we set

$$
\lambda=\theta\left(\frac{1}{p}-\frac{1}{q}\right), \alpha_{\theta}=1-\theta-\frac{1}{p_{\theta}}, a=\lambda-\theta, \lambda_{1}=\frac{1}{p_{\theta}}-\frac{1}{q}=(1-\theta)\left(\frac{1}{p}-\frac{1}{q}\right) .
$$

According to Theorem [5.2, the expression of the norm of $f$ in $W$ is composed with 3 terms:

$$
\|f\|_{W}^{r}=\int_{0}^{1}\left(t^{-\theta} K\left(f, t ; L^{(p}, L^{q)}\right)\right)^{r} \frac{d t}{t} \approx N_{1}+N_{2}+N_{3}
$$

with $N_{i}$ corresponding to the function $K_{i}$ and which reads as follow after a change of variables

$$
\begin{aligned}
& N_{1}=\int_{0}^{1}\left[t^{-\lambda}(1-\log t)^{a} \int_{0}^{t}(1-\log s)^{\frac{-1}{p}}\left(\int_{0}^{s} f_{*}^{p}(x) d x\right)^{\frac{1}{p}} \frac{d s}{s}\right]^{r} \frac{d t}{t} \\
& N_{2}=\int_{0}^{1}\left[t^{-\lambda}(1-\log t)^{\alpha_{\theta}}\left(\int_{0}^{t} f_{*}^{p}(x) d x\right)^{\frac{1}{p}}\right]^{r} \frac{d t}{t} \\
& N_{3}=\int_{0}^{1}\left[t^{\lambda_{1}}(1-\log t)^{\alpha_{\theta}+\frac{1}{q}} \sup _{t \leqslant s<1}(1-\log s)^{\frac{-1}{q}}\left(\int_{s}^{1} f_{*}^{q}(x) d x\right)^{\frac{1}{q}}\right]^{r} \frac{d t}{t} .
\end{aligned}
$$

We start with a lower bound for $\|f\|_{W}$.

\section{Lemma 5.1.}

One has

$$
\|f\|_{W}^{r} \gtrsim N_{3} \gtrsim\|f\|_{V}^{r}=\int_{0}^{1}\left[t^{\frac{1}{p_{\theta}}}(1-\log t)^{\alpha_{\theta}} f_{*}(t)\right]^{r} \frac{d t}{t} .
$$




\section{Proof:}

One has

$$
\begin{aligned}
N_{3} & \geqslant \int_{0}^{1}\left[t^{\lambda_{1}}(1-\log t)^{\alpha_{\theta}}\left(\int_{t}^{1} f_{*}^{q}(x) d x\right)^{\frac{1}{q}}\right]^{r} \frac{d t}{t} \\
& \geqslant \int_{0}^{\frac{1}{2}}\left[t^{\lambda_{1}}(1-\log t)^{\alpha_{\theta}}\left(\int_{t}^{2 t} f_{*}^{q}(x) d x\right)^{\frac{1}{q}}\right]^{r} \frac{d t}{t} \\
& \gtrsim \int_{0}^{\frac{1}{2}}\left[t^{\lambda_{1}+\frac{1}{q}}(1-\log t)^{\alpha_{\theta}} f_{*}(2 t)\right]^{r} \frac{d t}{t}
\end{aligned}
$$

Thus, we have, after a change of variables

$$
\int_{0}^{1}\left[t^{\frac{1}{p_{\theta}}}(1-\log t)^{\alpha_{\theta}} f_{*}(t)\right]^{r} \frac{d t}{t} \lesssim N_{3} \lesssim\|f\|_{W}^{r}
$$

For the upper bound, we start with the estimate of $N_{2}$

\section{Lemma 5.2.}

One has

$$
N_{2} \lesssim\|f\|_{V}^{r}
$$

\section{Proof:}

We set $\left.b=\frac{r}{p} \in\right] 0,+\infty\left[, \beta=p \alpha_{\theta}\right.$, we note that $0<\lambda p<1$ we can write

$$
N_{2}=\int_{0}^{1}\left[t^{-\lambda p}(1-\log t)^{\beta} \int_{0}^{t} f_{*}^{p}(x) d x\right]^{b} \frac{d t}{t} .
$$

We may apply the Hardy's inequalities of Theorem 2.1 to derive

$$
N_{2} \lesssim \int_{0}^{1}\left[t^{1-\lambda p}(1-\log t)^{\beta} f_{*}^{p}(t)\right]^{b} \frac{d t}{t} .
$$

The right hand side (RHS) of this inequality is equal to

$$
\int_{0}^{1}\left[t^{\frac{1}{p}-\lambda}(1-\log t)^{\alpha_{\theta}} f_{*}(t)\right]^{r} \frac{d t}{t}
$$

since $\frac{1}{p}-\lambda=\frac{1-\theta}{p}+\frac{\theta}{q}=\frac{1}{p_{\theta}}$, we get the result.

Next, we want to estimate $N_{1}$ 


\section{Lemma 5.3.}

One has:

$$
N_{1} \lesssim \int_{0}^{1}\left[t^{\frac{1}{p_{\theta}}}(1-\log t)^{\alpha_{\theta}-1} f_{*}(t)\right]^{r} \frac{d t}{t} \leqslant\|f\|_{V}^{r}
$$

Proof:

Let us set

$$
H(s)=s^{-1}(1-\log s)^{-\frac{1}{p}}\left(\int_{0}^{s} f_{*}^{p} d x\right)^{\frac{1}{p}} .
$$

Then, from the Hardy's inequality given in Theorem 2.1 and the expression of $N_{1}$ :

$$
N_{1} \lesssim \int_{0}^{1}\left[t^{1-\lambda}(1-\log t)^{a} H(t)\right]^{r} \frac{d t}{t}
$$

Replacing $H$, noticing that $a-\frac{1}{p}=-\theta-\frac{1}{p_{\theta}}=\alpha_{\theta}-1$, we then have from relation (37)

$$
N_{1} \lesssim \int_{0}^{1}\left[t^{-\lambda}(1-\log t)^{\alpha_{\theta}-1}\left(\int_{0}^{t} f_{*}^{p} d x\right)^{\frac{1}{p}}\right]^{r} \frac{d t}{t}
$$

Applying again the Hardy inequality as in Lemma 5.2 we have

$$
N_{1} \lesssim \int_{0}^{1}\left[t^{\frac{1}{p}-\lambda}(1-\log t)^{\alpha_{\theta}-1} f_{*}(t)\right]^{r} \frac{d t}{t}
$$

This gives the result.

It remains to estimate the term $N_{3}$. We have

\section{Lemma 5.4.}

One has

$$
N_{3} \lesssim\|f\|_{V}^{r}
$$

The key lemma to estimate $N_{3}$ is the analogous of Proposition 3.1

\section{Proposition 5.1.}

Let $K_{d}$ be a decreasing nonnegative function on $(0,1), \nu>0$ and $\beta$ two real numbers. Then

$$
\int_{0}^{1}\left[t^{\nu}(1-\log t)^{\beta} \sup _{t \leqslant s<1}(1-\log s)^{-\frac{1}{q}} K_{d}(s)\right]^{r} \frac{d t}{t} \approx \int_{0}^{1}\left[t^{\nu}(1-\log t)^{\beta-\frac{1}{q}} K_{d}(t)\right]^{r} \frac{d t}{t} .
$$


The proof follows the same argument as for Proposition 3.1.

Applying the Proposition 5.1, we deduce

$$
N_{3} \lesssim \int_{0}^{1}\left[t^{\lambda_{1}}(1-\log t)^{\alpha_{\theta}}\left(\int_{t}^{1} f_{*}^{q}(x) d x\right)^{\frac{1}{q}}\right]^{r} \frac{d t}{t}
$$

(The function $K_{d}(t)=\left(\int_{t}^{1} f_{*}^{q}(x) d x\right)^{\frac{1}{q}}$ is decreasing).

Again applying Hardy inequalities (see Theorem 2.1) we have

$$
N_{3} \lesssim \int_{0}^{1}\left[t^{\frac{1}{q}+\lambda_{1}}(1-\log t)^{\alpha_{\theta}} f_{*}(t)\right]^{r} \frac{d t}{t}
$$

But we have $\frac{1}{q}+\lambda_{1}=\frac{1}{p_{\theta}}$ so the RHS of the above equation (41) is the norm of $f$ in $V$ at the power $r$.

We have shown

$$
\|f\|_{W}^{r} \lesssim N_{1}+N_{2}+N_{3} \lesssim\|f\|_{V}^{r}
$$

and

$$
\|f\|_{W}^{r} \gtrsim N_{3} \gtrsim\|f\|_{V}^{r}
$$

This proves Theorem 5.1 .

\section{The critical case $p=q$. The interpolation space $\left(L^{p)}, L^{(p)}\right.$ and its $K$-functional}

The preceding study can be extended to the case where $p=q$. In this case, the inverse function of $\psi_{1}(x)=(1-\log x)^{-1}$, say $\varphi_{1}(t)=e^{1-\frac{1}{t}}$ will play the fundamental role to express the $K$-functional. Note that in this case we can't use the Lemma 2.1, we should do it it by direct calculation. .

Theorem 6.1. $K$-functional for $\left(L^{p)}, L^{(p)}\right.$

For $1<p<+\infty, 0<t<1, f \in L^{p)}+L^{(p}$, one has

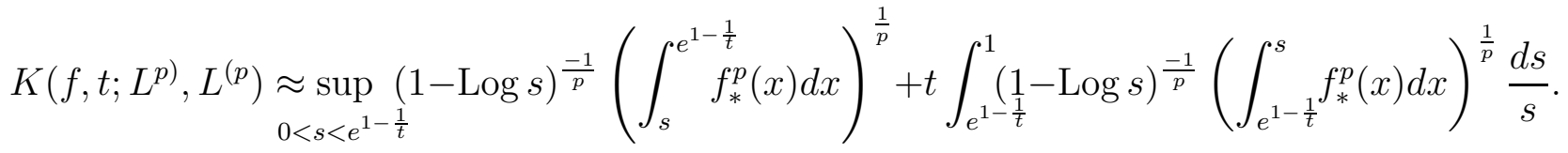




\section{Proof:}

We only sketch it since the arguments are similar to the proof of Theorem 3.2 .

We consider $f=g+h \in L^{p)}+L^{(p}$. Then for $t>0, f_{*}(t) \leqslant g_{*}\left(\frac{t}{2}\right)+h_{*}\left(\frac{t}{2}\right)$. We derive

$$
K^{0}(t)=\sup _{0<s<e^{1-\frac{1}{t}}}(1-\log s)^{\frac{-1}{p}}\left(\int_{s}^{e^{1-\frac{1}{t}}} f_{*}^{p}(x) d x\right)^{\frac{1}{p}} \leqslant I_{5}+I_{6}
$$

with

$$
I_{5}=\sup _{0<s<\varphi_{1}(t)}(1-\log s)^{\frac{-1}{p}}\left(\int_{s}^{\varphi_{1}(t)} g_{*}^{p}\left(\frac{x}{2}\right) d x\right)^{\frac{1}{p}} .
$$

With a change of variables, we have

$$
I_{5} \lesssim \sup _{0<s<\varphi_{1}(t)}\left(1-\log \frac{s}{2}\right)^{\frac{-1}{p}}\left(\int_{\frac{s}{2}}^{\varphi_{1}(t) / 2} g_{*}^{p}(x) d x\right)^{\frac{1}{p}} \lesssim\|g\|_{L^{p)}} .
$$

While for the term

$$
I_{6}=\sup _{0<s<\varphi_{1}(t)}(1-\log s)^{\frac{-1}{p}}\left(\int_{s}^{\varphi_{1}(t)} h_{*}^{p}\left(\frac{x}{2}\right) d x\right)^{\frac{1}{p}},
$$

since $1-\log \varphi_{1}(t)=\frac{1}{t}$ and $s \rightarrow(1-\log s)^{\frac{-1}{p}}$ is increasing, we have

$$
I_{6} \leqslant t^{\frac{1}{p}}\left(\int_{0}^{\varphi_{1}(t)} h_{*}^{p}(x) d x\right)^{\frac{1}{p}}=t \times t^{\frac{1}{p}-1}\left(\int_{0}^{\varphi_{1}(t)} h_{*}^{p}(x) d x\right)^{\frac{1}{p}} .
$$

We replace the quantity $t^{\frac{1}{p}-1}$ by making an integration of the following integral:

$$
\int_{\varphi_{1}(t)}^{1}(1-\log s)^{\frac{-1}{p}} \frac{d s}{s}=\frac{1}{1-\frac{1}{p}}\left[t^{\frac{1}{p}-1}-1\right] .
$$

Therefore, we have from (43) to (44)

$$
\begin{gathered}
I_{6} \lesssim t \int_{\varphi_{1}(t)}^{1}(1-\log s)^{\frac{-1}{p}}\left(\int_{0}^{s} h_{*}^{p}(x) d x\right)^{\frac{1}{p}} \frac{d s}{s}+t\|h\|_{L^{p}} \\
I_{6} \lesssim t\left(\|h\|_{L^{(p}}+\|h\|_{L^{p}}\right) \lesssim t\|h\|_{L^{(p}} .
\end{gathered}
$$

Thus, we have

$$
K^{0}(t) \lesssim\|g\|_{L^{p)}}+t|| h \|_{L^{(p}}
$$


While for the second term in the expression of $K$, say

$$
K^{1}(t)=t \int_{\varphi_{1}(t)}^{1}(1-\log s)^{\frac{-1}{p}}\left(\int_{\varphi_{1}(t)}^{s} f_{*}^{p}(x) d x\right)^{\frac{1}{p}} \frac{d s}{s} .
$$

One has

$$
K^{1}(t) \leqslant I_{7}+I_{8}
$$

with

$$
I_{7}=t \int_{\varphi_{1}(t)}^{1}(1-\log s)^{\frac{-1}{p}}\left(\int_{\varphi_{1}(t)}^{s} g_{*}^{p}\left(\frac{x}{2}\right) d x\right)^{\frac{1}{p}} \frac{d s}{s}
$$

and

$$
I_{8}=t \int_{e^{1-\frac{1}{t}}}^{1}(1-\log s)^{\frac{-1}{p}}\left(\int_{\varphi_{1}(t)}^{s} h_{*}^{p}\left(\frac{x}{2}\right) d x\right)^{\frac{1}{p}} \frac{d s}{s} .
$$

To estimate $I_{7}$, we use the relation $\frac{1}{t}=1-\log \varphi_{1}(t)$ to derive

$$
I_{7} \lesssim t\left(\int_{\varphi_{1}(t) / 2}^{1} g_{*}^{p}(x) d x\right)^{\frac{1}{p}} t^{\frac{1}{p}-1} \lesssim t\left(1-\log \frac{\varphi_{1}(t)}{2}\right)^{\frac{p-1}{p}}\left(\int_{\varphi_{1}(t) / 2}^{1} g_{*}^{p}(x) d x\right)^{\frac{1}{p}} \lesssim\|g\|_{L^{p)}}
$$

While for the second term $I_{8}$, we have after a change of variables

$$
I_{8} \lesssim t \int_{0}^{1}(1-\log x)^{\frac{-1}{p}}\left(\int_{0}^{x} h_{*}^{p}(\tau) d \tau\right)^{\frac{1}{p}} \frac{d x}{x}=t\|h\|_{L^{(p}}
$$

Then, we deduce from (48) to (49)

$$
K^{1}(t) \lesssim\|g\|_{L^{p)}}+t|| h \|_{L^{(p}}
$$

Therefore, we have for all $f=g+h$

$$
K^{0}(t)+K^{1}(t) \lesssim\|g\|_{L^{p)}}+t|| h \|_{L^{(p}}
$$

That is

$$
K^{0}(t)+K^{1}(t) \lesssim K\left(f, t ; L^{p)}, L^{(p)} .\right.
$$

For the reverse, we use the same decomposition as before $f \in L^{p)}+L^{(p}$, say

$$
g=\left(|f|-f_{*}\left(\varphi_{1}(t)\right)\right)_{+}=\left(|f|-f_{*}\left(\varphi_{1}(t)\right)\right) \chi_{\left\{|f|>f_{*}\left(\varphi_{1}(t)\right)\right\}}, \quad h=f-g,
$$

so that $h_{*}+g_{*}=f_{*}$ and

$$
g_{*}=\left(f_{*}-f_{*}\left(\varphi_{1}(t)\right)\right)_{+}, \quad h_{*}=f_{*}\left(\varphi_{1}(t)\right) \chi_{\left(0, \varphi_{1}(t)\right)}+f_{*}(s) \chi_{\left(\varphi_{1}(t), 1\right)} .
$$


With those expressions, we derive

$$
\|g\|_{L^{p)}} \leqslant \sup _{0<s<\varphi_{1}(t)}(1-\log s)^{\frac{1}{p}}\left(\int_{s}^{\varphi_{1}(t)} f_{*}^{p}(x) d x\right)^{\frac{1}{p}}=K^{0}(t) .
$$

While for the term in $h$,

$$
\begin{aligned}
t\|h\|_{L^{(p}} \leqslant & t\left(\int_{0}^{\varphi_{1}(t)}(1-\log s)^{\frac{-1}{p}} s^{\frac{1}{p}} \frac{d s}{s}\right) f_{*}\left(\varphi_{1}(t)\right) \\
& +t \int_{\varphi_{1}(t)}^{1}(1-\log s)^{\frac{-1}{p}} \frac{d s}{s} \varphi_{1}(t)^{\frac{1}{p}} f_{*}\left(\varphi_{1}(t)\right) \\
& +t \int_{\varphi_{1}(t)}^{1}(1-\log s)^{\frac{-1}{p}}\left(\int_{\varphi_{1}(t)}^{s} f_{*}^{p}(x) d x\right)^{\frac{1}{p}} \frac{d s}{s} .
\end{aligned}
$$

For the first integral in (53), one can use Proposition 2.2 to derive

$$
\int_{0}^{\varphi_{1}(t)} s^{\frac{1}{p}-1}(1-\log s)^{\frac{-1}{p}} d s \lesssim \varphi_{1}(t)^{\frac{1}{p}}\left(1-\log \varphi_{1}(t)\right)^{\frac{-1}{p}}
$$

So we obtain

$$
\begin{aligned}
t \int_{0}^{\varphi_{1}(t)}(1-\log s)^{\frac{-1}{p}} s^{\frac{1}{p}} \frac{d s}{s} f_{*}\left(\varphi_{1}(t)\right) & \lesssim t\left(1-\log \varphi_{1}(t)\right)^{\frac{-1}{p}}\left(\int_{\varphi_{1}(t) / 2}^{\varphi_{1}(t)} f_{*}^{p}(x) d x\right)^{\frac{1}{p}} \\
& \lesssim t \sup _{0<s \leqslant \frac{\varphi_{1}(t)}{2}}(1-\log s)^{\frac{-1}{p}}\left(\int_{s}^{\varphi_{1}(t)} f_{*}^{p}(x) d x\right)^{\frac{1}{p}} \\
& \lesssim t K^{0}(t) \leqslant K^{0}(t) .
\end{aligned}
$$

For the second integral, we use relation (44) to derive

$$
B=t \int_{\varphi_{1}(t)}^{1}(1-\log s)^{\frac{-1}{p}} \frac{d s}{s} \varphi_{1}(t)^{\frac{1}{p}} f_{*}\left(\varphi_{1}(t)\right) \lesssim t^{\frac{1}{p}} \varphi_{1}(t)^{\frac{1}{p}} f_{*}\left(\varphi_{1}(t)\right) .
$$

Since $\int_{e^{-\frac{1}{t}}}^{\varphi_{1}(t)} d x=\frac{e-1}{e} \varphi_{1}(t)$, we then have

$$
\varphi_{1}(t)^{\frac{1}{p}} f_{*}\left(\varphi_{1}(t)\right) \lesssim\left(\int_{e^{-\frac{1}{t}}}^{\varphi_{1}(t)} f_{*}^{p}(x) d x\right)^{\frac{1}{p}}
$$

So that relations (56) and (57) imply

$$
B=t \int_{\varphi_{1}(t)}^{1}(1-\log s)^{\frac{-1}{p}} \frac{d s}{s} \varphi_{1}(t)^{\frac{1}{p}} f_{*}\left(\varphi_{1}(t)\right) \lesssim\left(1-\log e^{1-\frac{1}{t}}\right)^{-\frac{1}{p}}\left(\int_{e^{-\frac{1}{t}}}^{\varphi_{1}(t)} f_{*}^{p}(x) d x\right)^{\frac{1}{p}} .
$$


Therefore, we obtain

$$
B \lesssim \sup _{0<s<\varphi_{1}(t)}(1-\log s)^{\frac{-1}{p}}\left(\int_{s}^{\varphi_{1}(t)} f_{*}^{p}(x) d x\right)^{\frac{1}{p}}=K^{0}(t) .
$$

The last term in relation (53) is equal to $K^{1}(t)$.

Combining these last relations (52) to (59), we come to

$$
\|g\|_{L^{p)}}+t|| h \|_{L^{(p}} \lesssim K^{0}(t)+K^{1}(t)
$$

which implies that

$$
K\left(f, t ; L^{p)}, L^{(p)} \lesssim K^{0}(t)+K^{1}(t) \lesssim K\left(f, t ; L^{p)}, L^{(p)} .\right.\right.
$$

We can now identify the interpolation space $\left(L^{p)}, L^{(p}\right)_{\theta, r}$ as we state in

\section{Theorem 6.2.}

Let $1<p<+\infty, 0<\theta<1,1 \leqslant r<+\infty$. Then $Z_{\theta, r} \doteq\left(L^{p)}, L^{(p}\right)_{\theta, r}$ has the following equivalent norm:

For $f \in Z_{\theta, r}, \beta_{\theta}=\theta-\frac{1}{p}-\frac{1}{r}$

- if $\theta<\frac{1}{p}$ then

$$
\|f\|_{Z_{\theta, r}} \approx\left[\int_{0}^{1}\left[(1-\log t)^{\beta_{\theta}}\left(\int_{t}^{1} f_{*}^{p}(s) d s\right)^{\frac{1}{p}}\right]^{r} \frac{d t}{t}\right]^{\frac{1}{r}}
$$

- if $\theta>\frac{1}{p}$ then $Z_{\theta, r}=G \Gamma(p, r, w), w(t)=t^{-1}(1-\log t)^{\beta_{\theta} r}$ and

$$
\|f\|_{Z_{\theta, r}} \approx\left[\int_{0}^{1}\left[(1-\log t)^{\beta_{\theta}}\left(\int_{0}^{t} f_{*}^{p}(s) d s\right)^{\frac{1}{p}}\right]^{r} \frac{d t}{t}\right]^{\frac{1}{r}}
$$

- if $\theta=\frac{1}{p}$

$$
\|f\|_{Z_{\theta, r}} \approx\left[\sum_{k=0}^{+\infty}\left(\int_{2^{1-2^{k+1}}}^{2^{1-2^{k}}} f_{*}^{p}(s) d s\right)^{\frac{r}{p}}\right]^{\frac{1}{r}}
$$

In particular

$$
\left(L^{p)}, L^{(p}\right)_{\frac{1}{p}, p}=L^{p} \quad \text { and } \quad\left(L^{p)}, L^{(p}\right)_{\theta, p}=L^{p, p}(\log L)^{\theta-\frac{1}{p}} .
$$




\section{Proof:}

According to Theorem 6.1, we have for $f \in Z_{\theta, r}$,

$$
\begin{aligned}
& \|f\|_{Z_{\theta, r}}^{r} \approx \int_{0}^{1}\left[t^{-\theta} \sup _{0<s<\varphi_{1}(t)}(1-\log s)^{\frac{-1}{p}}\left(\int_{s}^{\varphi_{1}(t)} f_{*}^{p}(y) d y\right)^{\frac{1}{p}}\right]^{r} \frac{d t}{t} \\
& +\int_{0}^{1}\left[t^{1-\theta} \int_{\varphi_{1}(t)}^{1}(1-\log s)^{\frac{-1}{p}}\left(\int_{\varphi_{1}(t)}^{s} f_{*}^{p}(y) d y\right)^{\frac{1}{p}} \frac{d s}{s}\right]^{r} \frac{d t}{t} \doteq F_{1}+F_{2} .
\end{aligned}
$$

Using the change of variables $x=\varphi_{1}(t)$ so that $t=(1-\log x)^{-1}$, we have

$$
\begin{gathered}
F_{1}=\int_{0}^{1}\left[(1-\log x)^{\theta} \sup _{0<s<x}(1-\log s)^{\frac{-1}{p}}\left(\int_{s}^{x} f_{*}^{p}(y) d y\right)^{\frac{1}{p}}\right]^{r} \frac{d x}{(1-\log x) x} \\
F_{2}=\int_{0}^{1}\left[(1-\log x)^{\theta-1} \int_{x}^{1}(1-\log s)^{\frac{-1}{p}}\left(\int_{x}^{s} f_{*}^{p}(y) d y\right)^{\frac{1}{p}} \frac{d s}{s}\right]^{r} \frac{d x}{(1-\log x) x} .
\end{gathered}
$$

Let us start with the upper bound of this norm.

\section{Proposition 6.1.}

One has

$$
F_{1}+F_{2} \lesssim \int_{0}^{1}\left[(1-\log x)^{\theta-\frac{1}{r}-\frac{1}{p}}\left(\int_{0}^{x} f_{*}^{p}(s) d s\right)^{\frac{1}{p}}\right]^{r} \frac{d x}{x}, \quad \text { if } \theta<1 .
$$

\section{Proof:}

For the term $F_{1}$, we have

$$
\left(\int_{s}^{x} f_{*}^{p}(t) d t\right)^{\frac{1}{p}} \leqslant\left(\int_{0}^{x} f_{*}^{p}(t) d t\right)^{\frac{1}{p}} \text { for } s<x
$$

so, one deduces

$$
F_{1} \leqslant \int_{0}^{1}\left[(1-\log x)^{\theta-\frac{1}{r}}\left(\int_{0}^{x} f_{*}^{p}(s) d s\right)^{\frac{1}{p}} \sup _{0<s<x}(1-\log s)^{\frac{-1}{p}}\right]^{r} \frac{d x}{x},
$$

which gives the upper estimate since $\sup _{0<s<x}(1-\log s)^{\frac{-1}{p}}=(1-\log x)^{\frac{-1}{p}}$.

For the term $F_{2}$, we have

$$
F_{2} \leqslant \int_{0}^{1}\left[(1-\log x)^{\theta-1-\frac{1}{r}} \int_{x}^{1}(1-\log s)^{\frac{-1}{p}}\left(\int_{0}^{s} f_{*}^{p}(y) d y\right)^{\frac{1}{p}} \frac{d s}{s}\right]^{r} \frac{d x}{x} .
$$


Since $\theta<1$, one may apply the Hardy inequality Theorem 2.2 (see [3] Theorem 6.5) to derive

$$
F_{2} \leqslant \int_{0}^{1}\left[(1-\log x)^{\theta-\frac{1}{r}-\frac{1}{p}}\left(\int_{0}^{x} f_{*}^{p}(y) d y\right)^{\frac{1}{p}}\right]^{r} \frac{d x}{x}
$$

\section{Proposition 6.2.}

For $0<\theta<1$, we have

$$
F_{1}+F_{2} \lesssim \int_{0}^{1}\left[(1-\log x)^{\theta-\frac{1}{r}-\frac{1}{p}}\left(\int_{x}^{1} f_{*}^{p}(y) d y\right)^{\frac{1}{p}}\right]^{r} \frac{d x}{x}
$$

\section{Proof:}

We start with $F_{2}$. Since

$$
\int_{x}^{s} f_{*}^{p}(y) d y \leqslant \int_{x}^{1} f_{*}^{p}(y) d y
$$

the expression of $F_{2}$ can be estimated as :

$$
F_{2} \leqslant \int_{0}^{1}\left[(1-\log x)^{\theta-1-\frac{1}{r}}\left(\int_{x}^{1} f_{*}^{p}(y) d y\right)^{\frac{1}{p}}\left(\int_{x}^{1}(1-\log s)^{-\frac{1}{p}} \frac{d s}{s}\right)\right]^{r} \frac{d x}{x} .
$$

By integration as for relation (44), one has

$$
\int_{x}^{1}(1-\log s)^{-\frac{1}{p}} \frac{d s}{s}=\frac{p}{p-1}\left[(1-\log x)^{1-\frac{1}{p}}-1\right] .
$$

Therefore, we deduce from (63) and (64), the

$$
F_{2} \lesssim \int_{0}^{1}\left[(1-\log x)^{\theta-\frac{1}{p}-\frac{1}{r}}\left(\int_{x}^{1} f_{*}^{p}(y) d y\right)^{\frac{1}{p}}\right]^{r} \frac{d x}{x}
$$

While for the second term $F_{1}$, we deduce from Bennett-Rudnick's Lemma ( [3] Lemma 6.1)

$$
\sup _{0<s<x}(1-\log s)^{-\frac{1}{p}}\left(\int_{s}^{1} f_{*}^{p}(y) d y\right)^{\frac{1}{p}} \leqslant c \int_{0}^{x}\left[(1-\log s)^{-\frac{1}{p}-1}\left(\int_{s}^{1} f_{*}^{p}(y) d y\right)^{\frac{1}{p}}\right] \frac{d s}{s} .
$$

Setting temporarily $\psi(s)=(1-\log s)^{-\frac{1}{p}-1}\left(\int_{s}^{1} f_{*}^{p}(y) d y\right)^{\frac{1}{p}}$, we then deduce

$$
F_{1} \lesssim \int_{0}^{1}\left[(1-\log x)^{\theta-\frac{1}{r}}\left(\int_{0}^{x} \psi(s) \frac{d s}{s}\right)\right]^{r} \frac{d x}{x}
$$


By Hardy inequality Theorem 2.2 (see [3] Theorem 6.5).

$$
F_{1} \lesssim \int_{0}^{1}\left[(1-\log x)^{\theta-\frac{1}{p}-\frac{1}{r}}\left(\int_{x}^{1} f_{*}^{p}(y) d y\right)^{\frac{1}{p}}\right]^{r} \frac{d x}{x}
$$

For the lower bound, we need few lemmas

The first lemma is a consequence of a general lemma given in Goldman Heinig and Stepanov ([20], see also [19] Lemma 3.1).

\section{Lemma 6.1.}

Let $t_{k}=2^{1-2^{k}}, \forall k \in \mathbb{N}, H$ a nonnegative locally integrable function on $(0,1),(q, \lambda) \in$ ] $0,+\infty\left[^{2}\right.$. Then

$$
\begin{aligned}
& \text { 1. } \sum_{k \in \mathbb{N}}\left(\int_{0}^{t_{k}} H(x) d x\right)^{q} 2^{\lambda k q} \approx \sum_{m \in \mathbb{N}}\left(2^{\lambda m} \int_{t_{m+1}}^{t_{m}} H(x) d x\right)^{q}, \\
& \text { 2. } \sum_{k \in \mathbb{N}}\left(\int_{t_{k+1}}^{1} H(x) d x\right)^{q} 2^{-\lambda k q} \approx \sum_{m \in \mathbb{N}}\left(2^{-\lambda m} \int_{t_{m+1}}^{t_{m}} H(x) d x\right)^{q} \text {. }
\end{aligned}
$$

Here $\mathbb{N}=\{0,1,2, \ldots\}$ the set of natural numbers.

The next Lemma can be obtained by straightforward computation

\section{Lemma 6.2.}

Let $t_{k}=2^{1-2^{k}}, k \in \mathbb{N}, \lambda \neq 0$. Then, one has

1. For any $s \in\left[t_{k+1}, t_{k}\right]$

$$
2^{k} \approx 1-\log s
$$

2.

$$
\int_{t_{k+1}}^{t_{k}}(1-\log t)^{\lambda-1} \frac{d t}{t} \approx 2^{k \lambda}
$$

for all $k \in \mathbb{N}$.

As a corollary of the above lemmas we have:

\section{Lemma 6.3.}

Let $t_{k}=2^{1-2^{k}}, k \in \mathbb{N}, \lambda \neq 0, q>0$ and $H$ a nonnegative locally integrable function on $(0,1)$. Then 
- If $\lambda>0$ then

$$
\sum_{k \in \mathbb{N}}\left(\int_{0}^{t_{k}} H(x) d x\right)^{q} 2^{\lambda k q} \gtrsim \int_{0}^{1}\left[(1-\log t)^{\lambda} \int_{0}^{t} H(x) d x\right]^{q} \frac{d t}{(1-\log t) t}
$$

and one has the equivalence if $\int_{0}^{1} H(x) d x \lesssim \int_{0}^{\frac{1}{2}} H(x) d x$ (for instance $H$ decreasing).

- If $\lambda<0$

$$
\sum_{k \in \mathbb{N}}\left(\int_{t_{k+1}}^{1} H(x) d x\right)^{q} 2^{\lambda k q} \approx \int_{0}^{1}\left[(1-\log t)^{\lambda} \int_{t}^{1} H(x) d x\right]^{q} \frac{d t}{(1-\log t) t}
$$

Proof:

If $\lambda>0$, we use statement 2.) of Lemma 6.2

$$
2^{\lambda k q} \approx \int_{t_{k+1}}^{t_{k}}(1-\log t)^{\lambda q-1} \frac{d t}{t}
$$

Thus, we have

$$
\sum_{k \in \mathbb{N}}\left(\int_{0}^{t_{k}} H(x) d x\right)^{q} 2^{\lambda k q} \approx \sum_{k \in \mathbb{N}}\left(\int_{0}^{t_{k}} H(x) d x\right)^{q} \int_{t_{k+1}}^{t_{k}}(1-\log t)^{\lambda q-1} \frac{d t}{t} .
$$

Since for $t<t_{k}, \int_{0}^{t} H(x) d x \leqslant \int_{0}^{t_{k}} H(x) d x$, then we derive from relation (67)

$$
\sum_{k \in \mathbb{N}}\left(\int_{0}^{t_{k}} H(x) d x\right)^{q} 2^{\lambda k q} \gtrsim \sum_{k \in \mathbb{N}} \int_{t_{k+1}}^{t_{k}}\left(\int_{0}^{t} H(x) d x\right)^{q}(1-\log t)^{\lambda q-1} \frac{d t}{t},
$$

and we have for any $G$ nonnegative

$$
\sum_{k \in \mathbb{N}} \int_{t_{k+1}}^{t_{k}} G(t) d t=\int_{0}^{1} G(t) d t
$$

This ends the proof of the lower bound of the first statement.

For the upper bound, we change the index of summation and use relation (66)

$$
\begin{aligned}
\sum_{k=1}^{+\infty}\left(\int_{0}^{t_{k}} H(x) d x\right)^{q} 2^{\lambda k q} & \approx \sum_{j \in \mathbb{N}}\left(\int_{0}^{t_{j+1}} H(x) d x\right)^{q} 2^{\lambda j q} \\
& \lesssim \sum_{j \in \mathbb{N}} \int_{t_{j+1}}^{t_{j}}\left(\int_{0}^{t} H(x) d x\right)^{q}(1-\log t)^{\lambda q-1} \frac{d t}{t} \\
& \lesssim \int_{0}^{1}(1-\log t)^{\lambda q-1}\left(\int_{0}^{t} H(x) d x\right)^{q} \frac{d t}{t}
\end{aligned}
$$


Moreover, if $\int_{0}^{1} H(x) d x \lesssim \int_{0}^{\frac{1}{2}} H(x) d x$ and since

$$
\begin{aligned}
\left(\int_{0}^{\frac{1}{2}} H(x) d x\right)^{q} & \lesssim \int_{\frac{1}{2}}^{1}\left(\int_{0}^{t} H(x) d x\right)^{q}(1-\log t)^{\lambda q-1} t^{-1} d t \\
& \lesssim \int_{0}^{1}\left[(1-\log t)^{\lambda} \int_{0}^{t} H(x) d x\right]^{q} \frac{d t}{(1-\log t) t}
\end{aligned}
$$

Then from the two relations (70) and (71), we obtain the upper bound for $\lambda>0$.

Same argument holds for $\lambda<0$ for having the second statement.

The above Lemma 6.3 holds for $\lambda=0$, noticing that $\int_{t_{k+1}}^{t_{k}} \frac{d t}{(1-\log t) t} \approx 1$. Then under the same conditions as before, we have

$$
\sum_{k \in \mathbb{N}}\left(\int_{0}^{t_{k}} H(x) d x\right)^{q} \approx \int_{0}^{1}\left[\int_{0}^{t} H(x) d x\right]^{q} \frac{d t}{(1-\log t) t}
$$

and

To obtain the lower bound for $F_{1}$, we first show the

$$
\sum_{k \in \mathbb{N}}\left(\int_{t_{k+1}}^{1} H(x) d x\right)^{q} \approx \int_{0}^{1}\left[\int_{t}^{1} H(x) d x\right]^{q} \frac{d t}{(1-\log t) t}
$$

\section{Lemma 6.4.}

One has

$$
F_{1} \gtrsim \sum_{k \in \mathbb{N}} 2^{k\left(\theta-\frac{1}{p}\right) r}\left(\int_{t_{k+1}}^{t_{k}} f_{*}^{p}(s) d s\right)^{\frac{r}{p}} \text { with } t_{k}=2^{1-2^{k}}, k \in \mathbb{N}
$$

Proof:

Let us set $G(x)=\sup _{0<s<x}(1-\log s)^{-\frac{r}{p}}\left(\int_{s}^{x} f_{*}^{p}(y) d y\right)^{\frac{r}{p}}, q=\frac{r}{p}$ and write

$$
F_{1}=\sum_{k \in \mathbb{N}} \int_{t_{k+1}}^{t_{k}} G(x)(1-\log x)^{\theta r-1} \frac{d x}{x} .
$$

Then, we derive from Lemma 6.2 and the definition of $G$

$$
F_{1} \gtrsim \sum_{k \in \mathbb{N}} G\left(t_{k}\right) \int_{t_{k+1}}^{t_{k}}(1-\log x)^{\theta r-1} \frac{d x}{x} \approx \sum_{k \in \mathbb{N}} G\left(t_{k}\right) 2^{k \theta r}
$$

But $G\left(t_{k}\right)=\sup _{i \geqslant k} \sup _{t_{i+1}<s<t_{i}}(1-\log s)^{-q}\left(\int_{s}^{t_{k}} f_{*}^{p}(y) d y\right)^{q}$ and Lemma 6.2 implies that for $t_{i+1}<s<t_{i}, \quad(1-\log s)^{-q} \approx 2^{-i q}$, so that

$$
G\left(t_{k}\right) \approx \sup _{i \geqslant k} 2^{-i q} \sup _{t_{i+1}<s<t_{i}}\left(\int_{s}^{t_{k}} f_{*}^{p}(y) d y\right)^{q},
$$


that is

$$
G\left(t_{k}\right) \approx \sup _{i \geqslant k} 2^{-i q}\left(\int_{t_{i+1}}^{t_{k}} f_{*}^{p}(y) d y\right)^{q}
$$

and then

$$
G\left(t_{k}\right) \approx \sup _{i \geqslant k} 2^{-i q}\left(\int_{t_{i+1}}^{t_{k}} f_{*}^{p}(y) d y\right)^{q} \geqslant 2^{-k q}\left(\int_{t_{k+1}}^{t_{k}} f_{*}^{p}(y) d y\right)^{q} .
$$

Combining relation (74) and (72), we deduce

$$
F_{1} \gtrsim \sum_{k \in \mathbb{N}} 2^{k \theta r-k \frac{r}{p}}\left(\int_{t_{k+1}}^{t_{k}} f_{*}^{p}(y) d y\right)^{\frac{r}{p}}
$$

This ends the proof.

As a corollary of the above lemmas, one has

\section{Theorem 6.3.}

On has for $f \in Z_{\theta, r}$

1. If $\theta>\frac{1}{p}$ then

$$
\|f\|_{Z_{\theta, r}}^{r} \gtrsim \int_{0}^{1}\left[(1-\log t)^{\theta-\frac{1}{p}}\left(\int_{0}^{t} f_{*}^{p}(x) d x\right)^{\frac{1}{p}}\right]^{r} \frac{d t}{(1-\log t) t} .
$$

2. If $\theta<\frac{1}{p}$ then

$$
\|f\|_{Z_{\theta, r}}^{r} \gtrsim \int_{0}^{1}\left[(1-\log t)^{\theta-\frac{1}{p}}\left(\int_{t}^{1} f_{*}^{p}(x) d x\right)^{\frac{1}{p}}\right]^{r} \frac{d t}{(1-\log t) t} .
$$

\section{Proof:}

Let us set $q=\frac{r}{p}, \lambda=\left|\theta-\frac{1}{p}\right| p$.

If $\theta-\frac{1}{p}>0$, we may apply statement 1 ) of Lemma 6.1 with $H(x)=f_{*}^{p}(x)$ to derive from Lemma 6.4

$$
F_{1} \gtrsim \sum_{k \in \mathbb{N}}\left(2^{\lambda k} \int_{t_{k+1}}^{t_{k}} f_{*}^{p}(y) d y\right)^{q} \approx \sum_{k \in \mathbb{N}}\left[\int_{0}^{t_{k}} f_{*}^{p}(x) d x\right]^{q} 2^{\lambda k q} .
$$

So that relation (75) gives with the help of Lemma 6.3

$$
F_{1} \gtrsim \sum_{k \in \mathbb{N}}\left(\int_{0}^{t_{k}} f_{*}^{p}(x) d x\right)^{q} 2^{\lambda k q} \gtrsim \int_{0}^{1}\left[(1-\log t)^{\lambda} \int_{0}^{t} f_{*}^{p}(x) d x\right]^{q} \frac{d t}{(1-\log t) t} .
$$


This last inequality implies the first statement of Theorem 6.3, noticing that

$$
\|f\|_{Z_{\theta, r}}^{r} \gtrsim F_{1}
$$

While for the second statement, we apply the statement 2) of Lemma 6.1 to derive from Lemma 6.4:

$$
F_{1} \gtrsim \sum_{k \in \mathbb{N}}\left(2^{-\lambda k} \int_{t_{k+1}}^{t_{k}} f_{*}^{p}(y) d y\right)^{q} \approx \sum_{k \in \mathbb{N}}\left[\int_{t_{k+1}}^{1} f_{*}^{p}(x) d x\right]^{q} 2^{-\lambda k q} .
$$

So that

$$
F_{1} \gtrsim \sum_{k \in \mathbb{N}}\left(\int_{t_{k+1}}^{1} f_{*}^{p}(x) d x\right)^{q} 2^{-\lambda k q} \gtrsim \int_{0}^{1}\left[(1-\log t)^{-\lambda} \int_{t}^{1} f_{*}^{p}(x) d x\right]^{q} \frac{d t}{(1-\log t) t} .
$$

The last inequality comes from the second statement of Lemma 6.3 and this ends the proof of Theorem 6.3 since $\|f\|_{Z_{\theta, r}}^{r} \gtrsim F_{1}$.

It remains to investigate in the particular case $\theta=\frac{1}{p}$. The lower bound for $\|f\|_{Z_{\theta, r}}$ comes from Lemma 6.4. It is sufficient to show the

\section{Lemma 6.5.}

For any $\theta \in] 0,1[$, we have

$$
\max \left(F_{1} ; F_{2}\right) \leqslant \sum_{k \in \mathbb{N}} 2^{k r\left(\theta-\frac{1}{p}\right)}\left(\int_{t_{k+1}}^{t_{k}} f_{*}^{p}(s) d s\right)^{\frac{r}{p}} .
$$

\section{Proof:}

The estimates for $F_{1}$ and $F_{2}$ follow the same argument, nevertheless we detail both estimates for clarity reason.

Following the notation in the proof of Lemma 6.4, we have

$$
F_{1}=\sum_{k \in \mathbb{N}} \int_{t_{k+1}}^{t_{k}} G(x)(1-\log x)^{\theta r-1} \frac{d x}{x} .
$$

Following the above argument as in Lemma 6.4, we have

$$
F_{1} \lesssim \sum_{k \in \mathbb{N}} G\left(t_{k}\right) 2^{k \theta r} \lesssim \sum_{k \in \mathbb{N}} 2^{k \theta r} \sup _{i \geqslant k} 2^{-i q}\left(\int_{t_{i+1}}^{t_{k}} f_{*}^{p}(y) d y\right)^{q}
$$

Writing

$$
\int_{t_{i+1}}^{t_{k}} f_{*}^{p}(y) d y=\sum_{j=k}^{i} 2^{j} 2^{-j} \int_{t_{j+1}}^{t_{j}} f_{*}^{p}(y) d y
$$


one deduces

$$
\sup _{i \geqslant k} 2^{-i q}\left(\int_{t_{i+1}}^{t_{k}} f_{*}^{p}(y) d y\right)^{q} \leqslant \sup _{i \geqslant k} 2^{-i q}\left(\sum_{j=k}^{i} 2^{j}\right)^{q} \sup _{j \geqslant k} 2^{-j q}\left(\int_{t_{j+1}}^{t_{j}} f_{*}^{p}(x) d x\right)^{q}
$$

Therefore, we have

$$
F_{1} \lesssim \sum_{k \in \mathbb{N}} 2^{k \theta r} \sup _{j \geqslant k} 2^{-j q}\left(\int_{t_{j+1}}^{t_{j}} f_{*}^{p}(x) d x\right)^{q}
$$

So, setting $A_{j} \doteq\left(\int_{t_{j+1}}^{t_{j}} f_{*}^{p}(y) d y\right)^{q}$ estimating the supremum term by a sum, one has

$$
\begin{gathered}
F_{1} \lesssim \sum_{k \in \mathbb{N}} 2^{k \theta r} \sum_{j=k}^{+\infty} 2^{-j q} A_{j}=\sum_{j \in \mathbb{N}} 2^{-j q} A_{j} \sum_{k=0}^{j} 2^{k \theta r} . \\
F_{1} \lesssim \sum_{j \in \mathbb{N}} 2^{j \theta r-j q} A_{j}=\sum_{j \in \mathbb{N}} 2^{j\left(\theta-\frac{1}{p}\right) r}\left(\int_{t_{j+1}}^{t_{j}} f_{*}^{p}(y) d y\right)^{q} .
\end{gathered}
$$

For estimating $F_{2}$, we set $G_{0}(x)=\left[\int_{x}^{1}(1-\log s)^{\frac{-1}{p}}\left(\int_{x}^{s} f_{*}^{p}(y) d y\right)^{\frac{1}{p}} \frac{d s}{s}\right]^{r}$ and write

$$
F_{2}=\sum_{k \in \mathbb{N}} \int_{t_{k+1}}^{t_{k}}(1-\log x)^{(\theta-1) r-1} G_{0}(x) \frac{d x}{x} .
$$

Then, using Lemma 6.2 and the fact that $G_{0}(x) \leqslant G_{0}\left(t_{k+1}\right)$ for $t_{k+1}<x<t_{k}$, one has

$$
F_{2} \lesssim \sum_{k \in \mathbb{N}} G_{0}\left(t_{k+1}\right) 2^{k r(\theta-1)}
$$

Writing $G_{0}\left(t_{k+1}\right)$ as

$$
G_{0}\left(t_{k+1}\right)=\left[\sum_{i=0}^{k} \int_{t_{i+1}}^{t_{i}}(1-\log s)^{\frac{-1}{p}}\left(\int_{t_{k+1}}^{s} f_{*}^{p}(y) d y\right)^{\frac{1}{p}} \frac{d s}{s}\right]^{r},
$$

and using Lemma 6.2, we then have

$$
G_{0}\left(t_{k+1}\right) \lesssim\left[\sum_{i=0}^{k}\left(\int_{t_{k+1}}^{t_{i}} f_{*}^{p}(y) d y\right)^{\frac{1}{p}} 2^{\left(1-\frac{1}{p}\right) i}\right]^{r}
$$


Using Lemma 6.1 with an adequate step function $H$, the RHS of relation (178) can be estimated as

$$
\begin{aligned}
\sum_{i=0}^{k}\left(\int_{t_{k+1}}^{t_{i}} f_{*}^{p}(y) d y\right)^{\frac{1}{p}} 2^{\left(1-\frac{1}{p}\right) i} & =\sum_{i=0}^{k} 2^{\left(1-\frac{1}{p}\right) i}\left(\sum_{j=i}^{k} \int_{t_{j+1}}^{t_{j}} f_{*}^{p}(y) d y\right)^{\frac{1}{p}} \\
& \approx \sum_{i=0}^{k} 2^{\left(1-\frac{1}{p}\right) i}\left(\int_{t_{i+1}}^{t_{i}} f_{*}^{p}(y) d y\right)^{\frac{1}{p}} .
\end{aligned}
$$

Summarizing the above relations, we come to

$$
F_{2} \lesssim \sum_{k \in \mathbb{N}} 2^{k r(\theta-1)}\left[\sum_{i=0}^{k} 2^{\left(1-\frac{1}{p}\right) i}\left(\int_{t_{i+1}}^{t_{i}} f_{*}^{p}(y) d y\right)^{\frac{1}{p}}\right]^{r}
$$

using the second statement of Lemma 6.1, by taking $H$ a step function on $[0,1]$ such that $\int_{t_{i+1}}^{t_{i}} H(x) d x=2^{\left(1-\frac{1}{p}\right) i}\left(\int_{t_{i+1}}^{t_{i}} f_{*}^{p}(y) d y\right)^{\frac{1}{p}}$, we deduce from this last inequality

$$
F_{2} \lesssim \sum_{i \in \mathbb{N}} 2^{i\left(\theta-\frac{1}{p}\right) r}\left(\int_{t_{i+1}}^{t_{i}} f_{*}^{p}(y) d y\right)^{\frac{r}{p}}
$$

This ends the proof.

\section{Corollary 6.1. of Lemma 6.5 and Lemma 6.4}

One has for any $\theta \in] 0,1\left[\right.$ and $f \in Z_{\theta, r}\|f\|_{Z_{\theta, r}}^{r} \approx \sum_{k \in \mathbb{N}} 2^{k r\left(\theta-\frac{1}{p}\right)}\left(\int_{t_{k+1}}^{t_{k}} f_{*}^{p}(y) d y\right)^{\frac{r}{p}}$.

As a consequence of the above corollary, we have

\section{Theorem 6.4.}

One has

$$
\|f\|_{Z_{\theta, r}} \approx\left[\int_{0}^{1}(1-\log t)^{\theta r}\left(\int_{0}^{t}(1-\log x)^{-1} f_{*}^{p}(x) d x\right)^{\frac{r}{p}} \frac{d t}{(1-\log t) t}\right]^{\frac{1}{r}} .
$$

\section{Proof:}

From the Lemma 6.2

$$
\int_{t_{k+1}}^{t_{k}} 2^{-k} f_{*}^{p}(y) d y \approx \int_{t_{k+1}}^{t_{k}}(1-\log y)^{-1} f_{*}^{p}(y) d y,
$$


and from the above Corollary 6.1

$$
\begin{aligned}
\|f\|_{Z_{\theta, r}}^{r} & \approx \sum_{k \in \mathbb{N}} 2^{k r \theta}\left(\int_{t_{k+1}}^{t_{k}}(1-\log x)^{-1} f_{*}^{p}(x) d x\right)^{\frac{r}{p}} \\
& \approx \sum_{k \in \mathbb{N}} 2^{k r \theta}\left(\int_{0}^{t_{k}}(1-\log x)^{-1} f_{*}^{p}(x) d x\right)^{\frac{r}{p}} \text { (using Lemma 6.1) } \\
& \approx \int_{0}^{1}(1-\log x)^{r \theta}\left(\int_{0}^{t}(1-\log t)^{-1} f_{*}^{p}(x) d x\right)^{\frac{r}{p}} \frac{d t}{(1-\log t) t} \text { (using Lemma 6.3) } .
\end{aligned}
$$

Acknowledgement : This paper has been written during the visit of the third author (A. Gogatishvili) at the University of Poitiers in April 2016; he wishes to thank all the members of the department for their kind hospitality.

The research of A. Gogatishvili and T.Kopaliani was in part supported by the Grant no DI/9/5-100/13 of the Shota Rustaveli National Science Foundation, and grant no 217282, Operators of Fourier analysis in some classical and new function spaces.

The research of A.Gogatishvili was partially supported by the grant P201/13/14743S of the Grant agency of the Czech Republic and RVO: 67985840.

The second author has been partially supported by the Gruppo Nazionale per l'Analisi Matematica, la Probabilità e le loro Applicazioni (GNAMPA) of the Istituto Nazionale di Alta Matematica (INdAM), by Project Legge 5/2007 Regione Campania "Spazi pesati ed applicazioni al calcolo delle variazioni" and by Università degli Studi di Napoli Parthenope through the project "sostegno alla Ricerca individuale" (triennio 2015-2017).

\section{References}

[1] I. Ahmed, D.E. Edmunds, W.D. Evans and G.E. Karadzhov, Reiteration theorems for the K-interpolation method in limiting cases. Math. Nachr. 284 (2011), 421-442.

[2] G. Anatriello, Iterated grand and small Lebesgue spaces, Collect. Math. 65 (2014), 273-284.

[3] C. Bennett, K. Rudnick, On Lorentz-Zygmund spaces Dissertationes Math. (Rozrrawy Mat.) 175 (1980) 67pp.

[4] J. Bastero and F. Ruiz, Elementary reverse Hölder type inequalities with application to operator interpolation theory, Proc. A.M.S. 124 (10) (1996), 3183-3192. 
[5] C. Bennett, R.Sharpley, Interpolation of Operators, Academic Press, 1988.

[6] J. Bergh, J. Löfstrom, Interpolation spaces. An introduction, Grundlehren der Mathematischen Wissenschaften, N²23, Springer-Verlag, Berlin-New-York, 1976.

[7] Yu. A. Brudnyi, N. Krugljak, Interpolation functors and interpolation spaces vol 1 Translated from Russian by N. Wadhwa. Mathematical Library 47 North-Holland Publishing, Amsterdam, 1991.

[8] C. Capone and A. Fiorenza On small Lebesgue spaces, J. Funct. Spaces Appl. 3 (2005), 73-89.

[9] F. Cobos, T. Kühn, Extrapolation results of Lions-Peetre type, Calc. Var. Partial Differential Equations, 49 (2014) 847-860.

[10] F. Cobos, O. Domínguez, Approximation spaces, limiting interpolation and Besov spaces. $J$. Approx. Theory 189 (2015) 43-66.

[11] W.D. Evans, B. Opic, L. Pick, Real interpolation with logarithmic functions J. Inequal. Appl. 7 (2002) 187-269.

[12] G. Di Fratta, A. Fiorenza, A direct approach in the duality of grand and small Lebesgue spaces. Nonlinear Anal. TMA 70 (2009) 2582-2592.

[13] P. Fernández-Martínez, T. Signes, Limit cases of reiteration theorems, Math. Nachr. 288 (2015) 25-47.

[14] A. Fiorenza, M.R. Formica, J.M. Rakotoson, Pointwise estimates for GГ-functions and applications, Differential and Integral equations 30, $\mathrm{N}^{\circ} 11-12$, (2017), 809-824.

[15] A. Fiorenza, G.E. Karadzhov Grand and small Lebesgue spaces and their analogs, Z. Anal. Anwendungen 23 (2004) 657-681.

[16] A. Fiorenza, J.M. Rakotoson, Compactness, interpolation inequalities for small LebesgueSobolev spaces and their applications, Calc. Var. Partial Differential Equations, 25 (2) (2005) $187-203$

[17] A. Fiorenza, J.M. Rakotoson, Some estimates in $G \Gamma(p, m, w)$ spaces, J. Math. Anal. Appl. 340 (2008) 793-805.

[18] A. Gogatishvili, B. Opic and W. Trebels, Limiting reiteration for real interpolation with slowly varying functions, Math. Nachr., 278 (2005), 86-107.

[19] A. Gogatishvili, L. Pick, Discretization and anti-discretization of rearrangement-invariant norms, Publ. Math. 47 (2003) 311-358.

[20] M.L. Gol'dman, H.P. Heinig, V.D. Stepanov, On the principle of duality in Lorentz spaces, Canad. J. Math. 48 (5) (1996) 959-979.

[21] B. Opic, A. Kufner, Hardy-type inequalities, Pitman Research Notes in Mathematics Series, Longman Scientific \& Technical, Harlow, 1990.

[22] L. Tartar, An introduction to Sobolev spaces and interpolation spaces, 2007, Springer. 